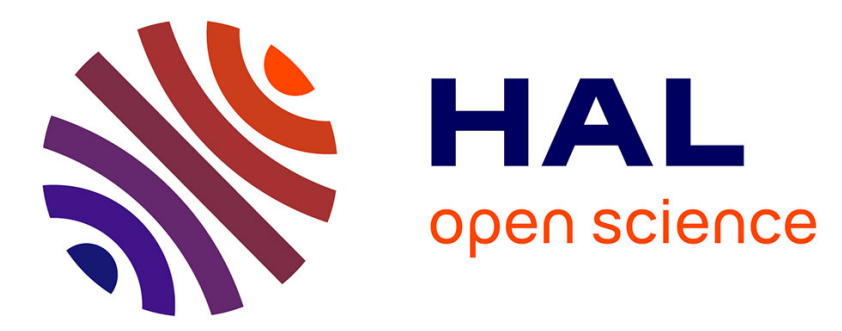

\title{
Ellipsoidal Anisotropies in Linear Elasticity: Extension of Saint Venant's Work to Phenomenological Modeling of Materials
}

Ahmad Pouya

\section{- To cite this version:}

Ahmad Pouya. Ellipsoidal Anisotropies in Linear Elasticity: Extension of Saint Venant's Work to Phenomenological Modeling of Materials. International Journal of Damage Mechanics, 2007, 16 (1), pp.95-126. 10.1177/1056789507065895 . hal-00571168

\section{HAL Id: hal-00571168 \\ https://hal.science/hal-00571168}

Submitted on 1 Mar 2011

HAL is a multi-disciplinary open access archive for the deposit and dissemination of scientific research documents, whether they are published or not. The documents may come from teaching and research institutions in France or abroad, or from public or private research centers.
L'archive ouverte pluridisciplinaire HAL, est destinée au dépôt et à la diffusion de documents scientifiques de niveau recherche, publiés ou non, émanant des établissements d'enseignement et de recherche français ou étrangers, des laboratoires publics ou privés. 


\title{
Ellipsoidal Anisotropies in Linear Elasticity: Extension of Saint Venant's Work to Phenomenological Modeling of Materials
}

\author{
Ahmad Pouya \\ LCPC - 58 Bd Lefebvre, 75732, Paris Cedex 15, France
}

\begin{abstract}
Several families of elastic anisotropies have been introduced by Saint Venant (Saint Venent, B. (de) (1863). Sur la distribution des élasticitiés autour de chaque point d'un solide ou d'un milieu de contexture quelconque, particulièrement lorsqu'il est amorphe sams être isotrope, Journal de Math. Pures et Appliquées, Tome VIII ( $2^{\text {éme }}$ série) pp. 257-430) for which the polar diagram of elastic parameters in different directions of the material (indicator surface) is ellipsoidal. These families cover a large variety of models introduced in recent years for damaged materials or as effective moduli of heterogeneous materials. Ellipsoidal anisotropy has also been used as a guideline in phenomenological modeling of materials. Then a question that naturally arises is to know in which conditions the assumption that some indicator surfaces are ellipsoidal allows one to entirely determine the elastic constants. This question has not been rigorously studied in the literature. In this study, first, several basic classes of ellipsoidal anisotropy are presented. Then the problem of the determination of elastic parameters from indicator surfaces is discussed in several basic cases that can occur in phenomenological modeling. Finally, the compatibility between the assumption of ellipsoidal form for different indicator surfaces is discussed. In particular, it is shown that if the indicator surfaces of $\sqrt[4]{E(\mathbf{n})}$ and of $\sqrt[-4]{c(\mathbf{n})}$ (where $E(\mathbf{n})$ and $c(\mathbf{n})$ are, respectively, the Young's modulus and the elastic coefficient in the direction $\mathbf{n}$ ) are ellipsoidal, then the two ellipsoids have necessarily the same principal axes, and the material in this case is orthotropic.
\end{abstract}

KEY WORDS: linear elasticity, anisotropy, indicator surface, ellipsoidal anisotropy, amorphous materials, damaged materials.

E-mail: pouya@1cpc.fr

Figures 1 and 2 appear in color online: http://ijd.sagepub.com

International Journal of DAMAge Mechanics, Vol. 16-January 2007 


\section{INTRODUCTION}

$\mathbf{O}$ $\mathrm{N}$ THE BASIS of the idea that the material isotropy geometrically corresponds to the image of a sphere, one can naturally seek to extend the isotropic models to anisotropic ones that correspond to an ellipsoidal variation of parameters in different directions. Saint Venant (1863) studied several elasticity models of this type, for instance, a model for which the polar diagram of $\sqrt[4]{E(\mathbf{n})}$, where $E(\mathbf{n})$ is the Young's modulus in direction $\mathbf{n}$, defines an ellipsoidal surface. He regarded these models as being useful for approximation of anisotropic elasticity of amorphous materials.

The models introduced by Saint Venant do not correspond to crystalline types of anisotropy, but cover a large variety of models introduced in recent years for the elasticity tensor of damaged materials (Halm and Dragon, 1988; Kachanov, 1992; Dragon et al., 2000; Chiarelli et al., 2003; Alliche, 2004) or as effective moduli of heterogeneous media (Milgrom and Shtrikman, 1992; Milton, 2002). They allow the representation of a three-dimensional anisotropy with reduced number of parameters.

In addition, the concept of ellipsoidal anisotropy has naturally been used as a guideline for modeling the elasticity of materials, or at least geomaterials such as soils, rocks, concrete, etc. The anisotropic behavior of these materials has extensively been studied in recent years by experimental or numerical methods and is taken into account in geotechnical design (Hefny and Lo, 1999; Pouya and Reiffsteck, 2003) and also in the study of seismic wave propagation, genesis of geological structures (Pan and Amadei, 1996), microcracking of rocks (Takemura et al., 2003), etc. Most of the times, a rough representation of the anisotropy with a minimum number of parameters is sufficient for the purposes of these studies. Peres Rodrigues and Aires-Barros (1970) tried to fit the measured values of the Young's modulus in different directions for several varieties of rocks by ellipsoids. For the study of seismic wave propagation in geological layers, Daley and Hron (1979) defined the 'elliptically anisotropic' medium as being characterized by elliptical P-wave fronts emanating from a point source. This concept was widely used in geophysical studies and examined by Thomsen (1986) in the context of weak anisotropy and transversal isotropy for a large variety of sedimentary rocks. Louis et al. (2004) proposed a simplified method to analyze the P-wave velocity data in anisotropic rocks, which supposes implicitly an ellipsoidal approximation of some elastic parameters. Pouya and Reiffsteck (2003) remarked that some 
Boehler's (1975) data on the Young's modulus of different soils presented an ellipsoidal property, and showed that this assumption allows one to simplify the modeling of foundations. As a matter of fact, it was shown by Pouya (2000) and Pouya and Zaoui (2005) that many closed form solutions for basic problems in linear isotropic materials can be extended by a linear transformation to various 'ellipsoidal' materials.

The concept of ellipsoidal anisotropy in elasticity thus seems an attractive guideline for phenomenological modeling of amorphous, microcracked, or damaged materials since it simplifies data analysis and defines models with a reduced number of parameters and interesting theoretical properties. Nevertheless, some theoretical questions concerning the existence and uniqueness of an elasticity tensor solution for one or more ellipsoidal indicator surfaces have not been examined rigorously in the literature. For instance, contrary to what was supposed by Peres Rodrigues and Aires-Barros (1970), the indicator surface of $E(\mathbf{n})$ can never be an ellipsoid (different from a sphere), and so the parameters fitted by this author do not define an elasticity tensor. In this article, first some elastic parameters which can have ellipsoidal variation are presented. Then the problem of determination of the elasticity tensor from indicator surfaces, which was studied by Hé and Curnier (1995) in restricted cases of damaged materials, are examined for basic cases of ellipsoidal surfaces.

\section{NOTATIONS}

In the following, light-face (Greek or Latin) letters denote scalars; bold-face minuscules and majuscules designate, respectively, vectors and second-rank tensors or double-index matrices; outline letters are reserved for fourth-rank tensors. The convention of summation on repeated indices is used implicitly. The scalar product of the two vectors is labeled as $\mathbf{a} \cdot \mathbf{b}=a_{i} b_{j}$, and their symmetric tensor product as $\mathbf{a} \otimes \mathbf{b}$ with $(\mathbf{a} \otimes \mathbf{b})_{i j}=\left(a_{i} b_{j}+a_{j} b_{i}\right) / 2$. The matrix product is labeled as $\mathbf{A B}$, and the inner product as $\mathbf{A}: \mathbf{B}=A_{i j} B_{i j}$. The operation of the fourth-rank tensor $\mathbb{C}$ on $\mathbf{A}$ is labeled by $\mathbb{C}: \mathbf{A}$ with $(\mathbb{C}: \mathbf{A})_{i j}=C_{i j k l} A_{k l}$ and the operation of $\mathbf{A}$ on a, by A.a.

For an elasticity tensor $\mathbb{C}$ verifying the symmetries $C_{i j k l}=C_{i j l k}=C_{k l i j}$, two distinct double index notations are introduced: the double subscript $(i j)$ is first abbreviated to a single subscript $(\alpha)$ running from 1 to 6 by the following rule:

$$
11 \rightarrow 1, \quad 22 \rightarrow 2, \quad 33 \rightarrow 3, \quad 23 \rightarrow 4, \quad 13 \rightarrow 5, \quad 12 \rightarrow 6
$$


The matrix notation $\boldsymbol{C}$ is defined by its components $\mathrm{c}_{\alpha \beta}=C_{(i j)(k l)}$, and the dual matrix notation $\underline{\boldsymbol{C}}$ for the same tensor $\mathbb{C}$, by its components $\mathrm{c}_{\alpha \beta}=\kappa c_{\alpha \beta}$ with

$$
\begin{array}{ll}
\kappa=1, & \text { if } \alpha \leq 3 \text { and } \beta \leq 3 \\
\kappa=4, & \text { if } \alpha>3 \text { and } \beta>3 \\
\kappa=2 & \text { elsewhere. }
\end{array}
$$

Note that the elastic compliances that are commonly designated in the literature by $s_{\alpha \beta}$ (Lekhnitskii, 1963; Sirotine and Chaskolskaia, 1984; Ting, 1996), are designated here by $s_{\alpha \beta}$.

\section{FOURTH-ORDER INDICATOR SURFACES}

The indicator surface for a 'monodirectional' elastic parameter, i.e., a parameter depending upon the elasticity tensor $\mathbb{C}$ and only one direction $\mathbf{n}$, is the polar diagram $\mathbf{x}=r(\mathbf{n}) \mathbf{n}$, where $\mathbf{n}$ is a unit vector and $r(\mathbf{n})$ is the value of the elastic parameter in the direction $\mathbf{n}$. Some examples of 'monodirectional' parameters are the Young's modulus, bulk modulus, elastic coefficient, and hydrostatic coefficient in direction $\mathbf{n}$ defined respectively by

$$
\begin{aligned}
E(\mathbf{n}) & =[(\mathbf{n} \otimes \mathbf{n}): \mathbb{S}:(\mathbf{n} \otimes \mathbf{n})]^{-1} \\
b(\mathbf{n}) & =[\delta: \mathbb{S}:(\mathbf{n} \otimes \mathbf{n})]^{-1} \\
c(\mathbf{n}) & =(\mathbf{n} \otimes \mathbf{n}): \mathbb{C}:(\mathbf{n} \otimes \mathbf{n}) \\
h(\mathbf{n}) & =\delta: \mathbb{C}:(\mathbf{n} \otimes \mathbf{n})
\end{aligned}
$$

where $\delta$ is the second-order unit tensor and $\mathbb{S}=\mathbb{C}^{-1}$. Other monodirectional parameters, such as the Poisson's ratio in direction $\mathbf{n}, v(\mathbf{n})=[1-E(\mathbf{n}) / b(\mathbf{n})] / 2$, or the torsion modulus in direction $\mathbf{n}, \tau(\mathbf{n})=\left[2\left(\delta_{i k}-n_{i} n_{k}\right) S_{i j k l} n_{j} n_{l}\right]^{-1}$, have also been introduced by some authors, and their indicator surfaces have been studied (Sirotine and Chaskolskaia, 1984). Investigation of this study is limited to the parameters defined by Equations (1)-(4), which are the basic parameters that can be determined experimentally from simple traction or extension tests.

The indicator surface of Young's modulus has been widely studied for all types of materials. The polar equation of this surface:

$$
r(\mathbf{n})=E(\mathbf{n})=[(\mathbf{n} \otimes \mathbf{n}): \mathbb{S}:(\mathbf{n} \otimes \mathbf{n})]^{-1}
$$


can be transformed, by using $r^{2}=\mathbf{x} \cdot \mathbf{x}$ and $\mathbf{n}=\mathbf{x} / r$, into the following polynomial equation:

$$
[(\mathbf{x} \otimes \mathbf{x}): \mathbb{S}:(\mathbf{x} \otimes \mathbf{x})]^{2}=(\mathbf{x} \cdot \mathbf{x})^{3}
$$

This eighth-order surface has been, in particular, deeply investigated by Cazzani and Marco $(2003,2005)$ for cubic, hexagonal, and tetragonal symmetries. It takes, in general, very complex forms (see Figure 1) and further analysis has shown that it cannot be an ellipsoid different from a sphere (Appendix 1). So this surface is not suitable for an ellipsoidal approximation of $E(\mathbf{n})$ values contrarily to that supposed by Peres Rodrigues (1970). On the other hand, if the indicator surface of $\sqrt[4]{E(\mathbf{n})}$ is considered then a fourth-order surface is found which is described by the following equation:

$$
(\mathbf{x} \otimes \mathbf{x}): \mathbb{S}:(\mathbf{x} \otimes \mathbf{x})=1
$$

This surface, which contains exactly the same information as that of the previous one, degenerates for some cases of materials to second-order surfaces, and more precisely, to ellipsoids. This defines a class of materials that are said to have a variety of ellipsoidal anisotropy.

Other fourth-order indicator surfaces which can degenerate to ellipsoids are those of $\sqrt{E(\mathbf{n})},[c(\mathbf{n})]^{-1 / 4}$, and $[c(\mathbf{n})]^{-1 / 2}$. The indicator surfaces of $[b(\mathbf{n})]^{1 / 2}$ and $[h(\mathbf{n})]^{-1 / 2}$ are always ellipsoidal. The denomination and equations of these surfaces are given in Table 1.

\section{SAINT VENANT'S ANISOTROPIES}

Saint Venant (1863) introduced several families of orthotropic materials for which one or more of the surfaces $F_{4}(\mathbb{C}), G_{4}(\mathbb{C}), F_{2}(\mathbb{C})$, or $G_{2}(\mathbb{C})$ are ellipsoidal. Let $\mathcal{B}=\left\{\mathbf{e}_{1}, \mathbf{e}_{2}, \mathbf{e}_{2}\right\}$ be a set of unit vectors defining a Cartesian coordinate system, and $\Omega(\mathcal{B})$ be the family of orthotropic materials with planes of symmetry given by $\mathcal{B}$.

The first family defined by Saint Venant is the subset of $\Omega(\mathcal{B})$, denoted here by $\Phi_{4}(\mathcal{B})$, for which the following relations between the parameters are satisfied:

$$
c_{44}=\frac{\sqrt{c_{22} c_{33}}-c_{23}}{2}, \quad c_{55}=\frac{\sqrt{c_{11} c_{33}}-c_{13}}{2}, \quad c_{66}=\frac{\sqrt{c_{11} c_{22}}-c_{12}}{2}
$$




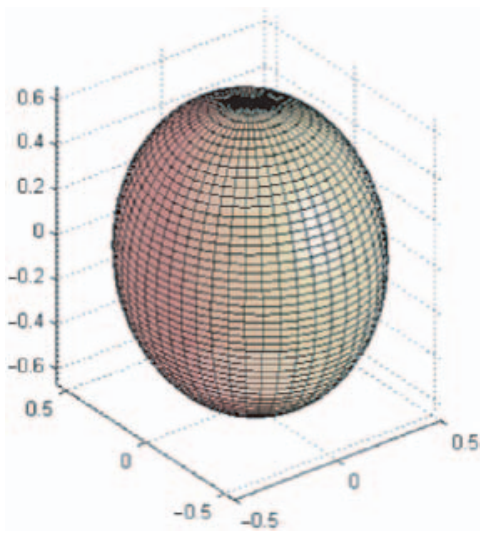

(a)

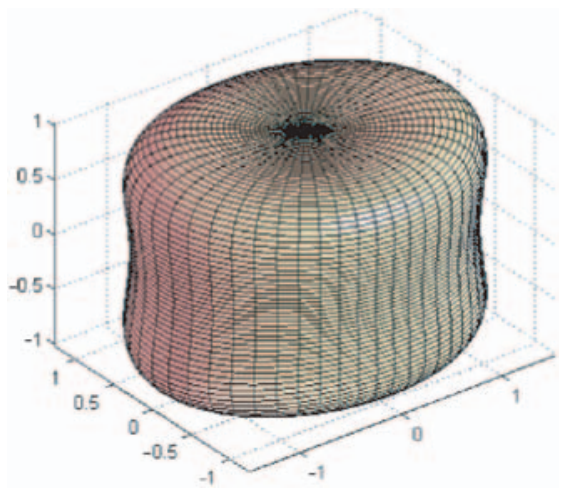

(c)

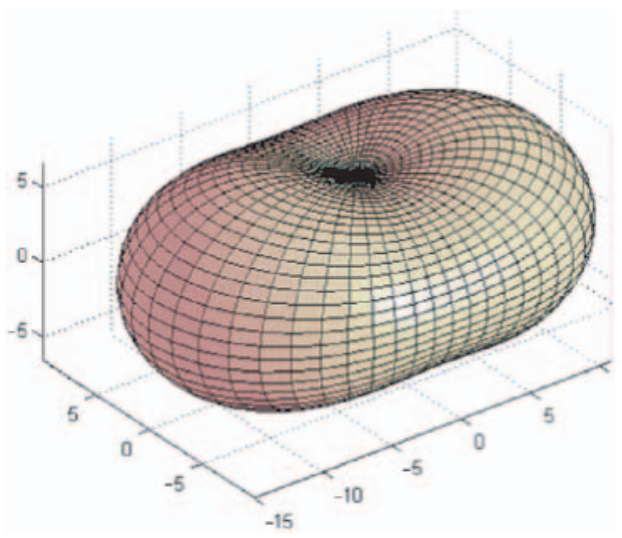

(b)

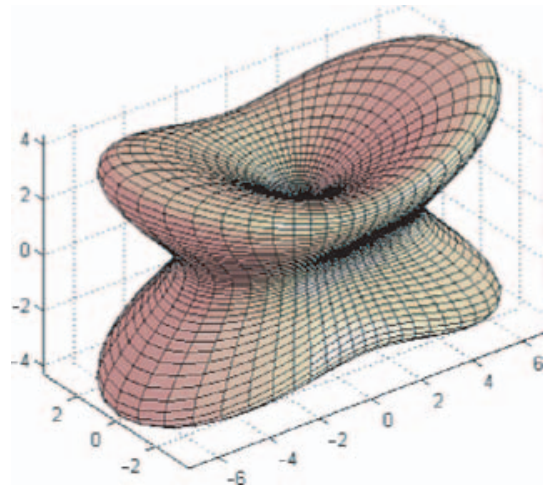

(d)

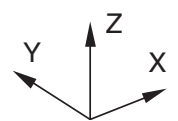

Figure 1. Indicator surfaces for an element of $\Phi_{4}$. For $\mathbb{C} \in \Phi_{4}(B)$ with $c_{11}=15, c_{22}=10$, $c_{33}=5, c_{12}=-3, c_{13}=4, c_{23}=4.5$, and $c_{44}, c_{55}, c_{66}$ given by Equation (8), the indicator surfaces of $\sqrt[-4]{c(n)}, c(n), \sqrt[4]{E(n)}$, and $E(n)$ are respectively (a) an ellipsoid, (b) a 10th-order, (c) 4th-order and (d) 8th-order surface.

For $\mathbb{C} \in \Phi_{4}(\mathcal{B})$, the equation of $F_{4}(\mathbb{C})$, given in Table 1 , reduces to the equation of an ellipsoid with principal axes $\mathcal{B}$ and semi-diameters $\left\{\left(c_{11}\right)^{-1 / 4},\left(c_{22}\right)^{-1 / 4},\left(c_{33}\right)^{-1 / 4}\right\}$ :

$$
\sqrt{c_{11}} x_{1}^{2}+\sqrt{c_{22}} x_{2}^{2}+\sqrt{c_{33}} x_{3}^{2}=1
$$


Table 1. Indicator surfaces of different elastic parameters and their polynomial equation.

\begin{tabular}{lll}
\hline Indicator surface & \multicolumn{1}{c}{ Elastic parameter } & \multicolumn{1}{c}{ Equation } \\
\hline$F_{4}(\mathbb{C})$ & {$[(\mathbf{n} \otimes \mathbf{n}): \mathbb{C}:(\mathbf{n} \otimes \mathbf{n})]^{-1 / 4}$} & $(\mathbf{x} \otimes \mathbf{x}): \mathbb{C}:(\mathbf{x} \otimes \mathbf{x})=1$ \\
$F_{2}(\mathbb{C})$ & {$[(\mathbf{n} \otimes \mathbf{n}): \mathbb{C}:(\mathbf{n} \otimes \mathbf{n})]^{-1 / 2}$} & $(\mathbf{x} \otimes \mathbf{x}): \mathbb{C}:(\mathbf{x} \otimes \mathbf{x})=\mathbf{x} \cdot \mathbf{x}$ \\
$G_{4}(\mathbb{C})$ & {$[(\mathbf{n} \otimes \mathbf{n}): \mathbb{S}:(\mathbf{n} \otimes \mathbf{n})]^{-1 / 4}$} & $(\mathbf{x} \otimes \mathbf{x}): \mathbb{S}:(\mathbf{x} \otimes \mathbf{x})=1$ \\
$G_{2}(\mathbb{C})$ & {$[(\mathbf{n} \otimes \mathbf{n}): \mathbb{S}:(\mathbf{n} \otimes \mathbf{n})]^{-1 / 2}$} & $(\mathbf{x} \otimes \mathbf{x}): \mathbb{S}:(\mathbf{x} \otimes \mathbf{x})=\mathbf{x} \cdot \mathbf{x}$ \\
$\mathrm{f}(\mathbb{C})$ & {$[\boldsymbol{\delta}: \mathbb{C}:(\mathbf{n} \otimes \mathbf{n})]^{-1 / 2}$} & $\boldsymbol{\delta}: \mathbb{C}:(\mathbf{x} \otimes \mathbf{x})=1$ \\
$\mathrm{~g}(\mathbb{C})$ & {$[\boldsymbol{\delta}: \mathbb{S}:(\mathbf{n} \otimes \mathbf{n})]^{-1 / 2}$} & $\boldsymbol{\delta}: \mathbb{S}:(\mathbf{x} \otimes \mathbf{x})=1$ \\
\hline
\end{tabular}

Equations (8) were considered by many authors, in the context of transversal isotropy, as defining a degenerate case (Pan and Chou, 1976).

The second family is that of orthotropic tensors for which relations analogous to Equation (8) hold between the parameters $s_{\alpha \beta}$. In terms of $s_{\alpha \beta}$, these relations become:

$$
\underline{S}_{44}=2\left(\sqrt{\underline{s}_{22} \underline{S}_{33}}-\underline{s}_{23}\right), \quad \underline{S}_{55}=2\left(\sqrt{\underline{S}_{11} \underline{S}_{33}}-\underline{S}_{13}\right), \quad \underline{S}_{66}=2\left(\sqrt{\underline{S}_{11} \underline{S}_{22}}-\underline{S}_{12}\right)
$$

and in terms of elastic modulus and Poisson's ratio (Equation (35)), they become:

$$
G_{23}=\frac{\sqrt{E_{2} E_{3}}}{2\left(1+\sqrt{\nu_{23} v_{32}}\right)}, \quad G_{31}=\frac{\sqrt{E_{3} E_{1}}}{2\left(1+\sqrt{\nu_{31} v_{13}}\right)}, \quad G_{12}=\frac{\sqrt{E_{1} E_{2}}}{2\left(1+\sqrt{\nu_{12} v_{21}}\right)}
$$

This family, which was also quoted by Lekhnitskii (1963), is labeled here as $\Gamma_{4}(\mathcal{B})$. For $\mathbb{C} \in \Gamma_{4}(\mathcal{B})$, the surface $G_{4}(\mathbb{C})$ is an ellipsoid with principal axes $\mathcal{B}$ and semidiameters $\left\{\left(s_{11}\right)^{-1 / 4},\left(s_{22}\right)^{-1 / 4},\left(s_{33}\right)^{-1 / 4}\right\}$, or $\left\{\sqrt[4]{E_{1}}, \sqrt[4]{E_{2}}, \sqrt[4]{E_{3}}\right\}$. In the context of transversal isotropy (with axis $x_{3}$ ), the third equality of Equation (10) is always satisfied and the first two are equivalent. In this context, these relations were considered by Boehler (1982) as defining a case of limited anisotropy with elliptical properties.

The elements of $\Phi_{4}(\mathcal{B})$ and $\Gamma_{4}(\mathcal{B})$ depend on six intrinsic parameters. The intersection between $\Phi_{4}(\mathcal{B})$ and $\Gamma_{4}(\mathcal{B})$ is denoted by $\Psi(\mathcal{B})$ :

$$
\Psi(\mathcal{B})=\Phi_{4}(\mathcal{B}) \cap \Gamma_{4}(\mathcal{B})
$$


This family of materials, as shown by Saint Venant, depends on four intrinsic parameters in the coordinates system $\mathcal{B}$, and can be represented equivalently by one of the following expressions of $\boldsymbol{C}$ or of $\underline{S}$ in this coordinates system:

$\Psi(\mathcal{B})$ :

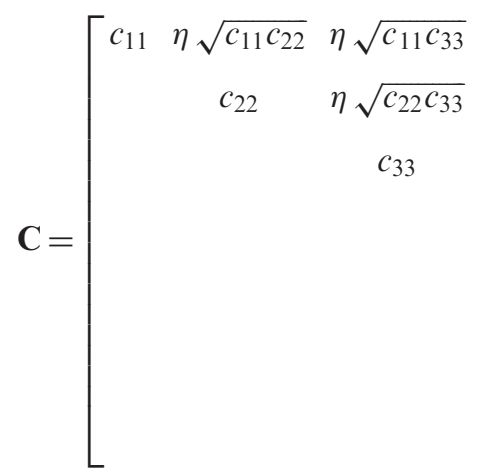

$$
\left.\begin{array}{lll}
\frac{1-\eta}{2} \sqrt{c_{22} c_{33}} & & \\
& \frac{1-\eta}{2} \sqrt{c_{11} c_{33}} & \\
& \frac{1-\eta}{2} \sqrt{c_{11} c_{22}}
\end{array}\right]
$$

$\Psi(\mathcal{B}): \quad \underline{\boldsymbol{S}}=\left[\begin{array}{ccccc}\frac{1}{E_{1}} & \frac{-v}{\sqrt{E_{1} E_{2}}} & \frac{-v}{\sqrt{E_{1} E_{3}}} & & \\ \frac{-v}{\sqrt{E_{1} E_{2}}} & \frac{1}{E_{2}} & \frac{-v}{\sqrt{E_{2} E_{3}}} & & \\ \frac{-v}{\sqrt{E_{1} E_{3}}} & \frac{-v}{\sqrt{E_{2} E_{3}}} & \frac{1}{E_{3}} & & \\ & & \frac{2(1+v)}{\sqrt{E_{2} E_{3}}} & \\ & & \frac{2(1+v)}{\sqrt{E_{3} E_{1}}} & \\ & & & \frac{2(1+v)}{\sqrt{E_{1} E_{2}}}\end{array}\right]$

The two sets of parameters $\left(\mathrm{c}_{11}, \mathrm{c}_{22}, \mathrm{c}_{33}, \eta\right)$ and $\left(E_{1}, E_{2}, E_{3}, v\right)$ are related by:

$v=\frac{\eta}{1+\eta}, \quad E_{\alpha}=\frac{(1-\eta)(1+2 \eta)}{1+\eta} c_{\alpha \alpha} \quad(\alpha=1,2,3 ;$ non-summation upon $\alpha)$ 
Pouya and Zaoui (2005) have shown that for this family of materials, $\mathbb{C}$ can be written in the following forms:

$$
\begin{aligned}
C_{i j k l} & =P_{i m} P_{j n} P_{k p} P_{l q} \tilde{C}_{m n p q} \\
C_{i j k l} & =\lambda D_{i j} D_{k l}+\mu\left(D_{i k} D_{j l}+D_{i l} D_{j k}\right)
\end{aligned}
$$

In these relations $\tilde{\mathbb{C}}$ represents an isotropic elasticity tensor with Lamé constants $\lambda=\eta$ and $\mu=(1-\eta) / 2, \mathbf{P}$ is given in the coordinates system $\mathcal{B}$ by

$$
\mathbf{P}=\operatorname{diag}\left(\sqrt[4]{c_{11}}, \quad \sqrt[4]{c_{22}}, \quad \sqrt[4]{c_{33}}\right)
$$

and $\mathbf{D}=\mathbf{P P}$.

Conversely, it can be checked that for the materials defined by Equation (17), both $\mathrm{F}_{4}(\mathbb{C})$ and $\mathrm{G}_{4}(\mathbb{C})$ are ellipsoidal. Therefore, Equation (17) defines $\Psi$ type materials where:

$$
\Psi=\bigcup_{\mathcal{B}} \Psi(\mathcal{B})
$$

The expression (17) has been used in some micromechanical studies as representing the effective modulus of heterogeneous media (Milgrom and Shtrikman, 1992, Milton, 2002). $\Psi$-type materials present interesting theoretical properties. Saint Venant showed that D'Alembert's displacement potentials as well as the solution for plane waves propagation in isotropic elasticity can be extended to this family. Pouya (2000) and Pouya and Zaoui (2005) showed that Equation (16) between the two elasticity tensors allows one to extend many closed form solutions for basic problems of elasticity to $\Psi$-type materials. Some examples of results extended in this way to $\Psi$-type materials are the Eshelby tensor for inclusion-matrix problem (Milgrom and Shtrikman, 1992; Pouya, 2000) and Green function for infinite space (Pouya, 2000) and for half-space (Pouya and Zaoui, 2005). The extension of the Green function solutions for two joined semi-infinite isotropic solids (Rongved, 1955) or for layered medium constituted of isotropic materials (Benitez and Rosakis, 1987) to solids constituted of $\Psi$-type materials would also be possible (Pouya and Zaoui, 2005).

Thus, theoretical simplifications can result from choosing $\Psi$-type models for representing (as an approximation) the elastic anisotropy of materials.

Now Equation (17) is considered. The tensor $\mathbf{D}$ can be decomposed as $\mathbf{D}=p \boldsymbol{\delta}+\alpha \mathbf{G}$ where $p=(\mathbf{D}: \boldsymbol{\delta}) / 3$ and $\mathbf{G}$ is traceless and normalized to unity: $\mathbf{G}: \boldsymbol{\delta}=0, \mathbf{G}: \mathbf{G}=1$. The parameter $p$ can be chosen with $\lambda$ and $\mu$ : consider $p=1$ without loss of generality and write $\mathbf{D}=\boldsymbol{\delta}+\alpha \mathbf{G}$. If $\alpha=0$, then Equation (17) gives the isotropic elasticity tensor. In the context of 
'weak anisotropy', i.e., when $|\alpha| \ll 1$, the first-order expansion of Equation (17) with respect to $\alpha$ leads to:

$$
\begin{aligned}
C_{i j k l}= & \lambda \delta_{i j} \delta_{k l}+\mu\left(\delta_{i k} \delta_{j l}+\delta_{i l} \delta_{j k}\right)+a_{1}\left(\delta_{i j} G_{k l}+\delta_{k l} G_{i j}\right) \\
& +a_{2}\left(\delta_{i k} G_{j l}+\delta_{i l} G_{j k}+\delta_{j l} G_{i k}+\delta_{j k} G_{i l}\right)
\end{aligned}
$$

with $a_{1}=\lambda \alpha$ and $a_{2}=\mu \alpha$. Equation (20) with independent values for $a_{1}$ and $a_{2}$ (and not necessarily infinitesimal) has been widely used in the literature for representing the elasticity tensor of damaged materials. It has been obtained by Kachanov (1992) as the effective moduli of microcracked media and then widely used as a phenomenological model for damaged geomaterials (Chiarelli et al., 2003; Alliche, 2004) or as an intermediary between micromechanical and phenomenological models for further theoretical investigations (Halm and Dragon, 1988; Dragon et al., 2000).

It is interesting to note that Equation (20) can be defined directly by an ellipsoidal property: for this model the surface $F_{2}(\mathbb{C})$ is ellipsoidal. Saint Venant defined a subset of $\Omega(\mathcal{B})$, denoted here by $\Phi_{2}(\mathcal{B})$, for which the following relations are satisfied between the parameters:

$$
c_{44}=\frac{c_{22}+c_{33}-2 c_{23}}{4}, \quad c_{55}=\frac{c_{11}+c_{33}-2 c_{13}}{4}, \quad c_{66}=\frac{c_{11}+c_{22}-2 c_{12}}{4}
$$

For $\mathbb{C} \in \Phi_{2}(\mathcal{B})$, the surface $F_{2}(\mathbb{C})$ is an ellipsoid with principal axes $\mathcal{B}$ and semidiameters $\left(1 / \sqrt{c_{11}}, 1 / \sqrt{c_{22}}, 1 / \sqrt{c_{33}}\right)$. The elements of this family have the Equation (20) where $\mathbf{G}$ is diagonal in $\mathcal{B}$. It can be checked that Equation (20) defines exactly the family $\Phi_{2}$ of materials given by:

$$
\Phi_{2}=\bigcup_{\mathcal{B}} \Phi_{2}(\mathcal{B})
$$

Finally, a subfamily of $\Omega(\mathcal{B})$, denoted here by $\Gamma_{2}(\mathcal{B})$, was defined by Saint Venant for which an Equation (21)-type relation is verified between $\mathrm{s}_{\alpha \beta}$ parameters, or equivalently (see also Lekhnitskii, 1963):

$$
\begin{aligned}
& \frac{1}{G_{23}}-\frac{2 \sqrt{\nu_{23} \nu_{32}}}{\sqrt{E_{2} E_{3}}}=\frac{1}{E_{2}}+\frac{1}{E_{3}}, \quad \frac{1}{G_{31}}-\frac{2 \sqrt{\nu_{31} \nu_{13}}}{\sqrt{E_{3} E_{1}}}=\frac{1}{E_{3}}+\frac{1}{E_{1}}, \\
& \frac{1}{G_{12}}-\frac{2 \sqrt{\nu_{12} \nu_{21}}}{\sqrt{E_{1} E_{2}}}=\frac{1}{E_{1}}+\frac{1}{E_{2}}
\end{aligned}
$$

For this family, $G_{2}(\mathbb{C})$ is ellipsoidal with semidiameters $\left\{\sqrt{E_{1}}, \sqrt{E_{2}}, \sqrt{E_{3}}\right\}$. 


\section{CHARACTERIZATION OF THE MATERIAL BY INDICATOR SURFACES}

For the families studied here previously, the expression of the tensors $\mathbb{C}$ or $\mathbb{S}$ is given a priori. Now consider that the values of some monodirectional elastic parameters have been determined in different directions by experimental or numerical methods. $E(\mathbf{n})$ values have frequently been determined experimentally by coring samples in different directions in rocks and soils (Boehler, 1975). Numerical homogenization methods allow one to easily determine $c(\mathbf{n})$ or $E(\mathbf{n})$ in different directions. For instance, Min and Jing (2003) determined the $E(\mathbf{n})$ values for a fractured rock mass by applying a compression parallel to the sides of a square REV and by rotating the REV with respect to the fractures (Figure 2). Acoustic measurements are widely used for determining the parameters of anisotropic elasticity in different directions. Sometimes a complete set of numerical values is determined by this method for the 21 parameters of general anisotropy (Homand et al., 1993). In this case it is interesting to derive an approximate model having a reduced number of parameters and,

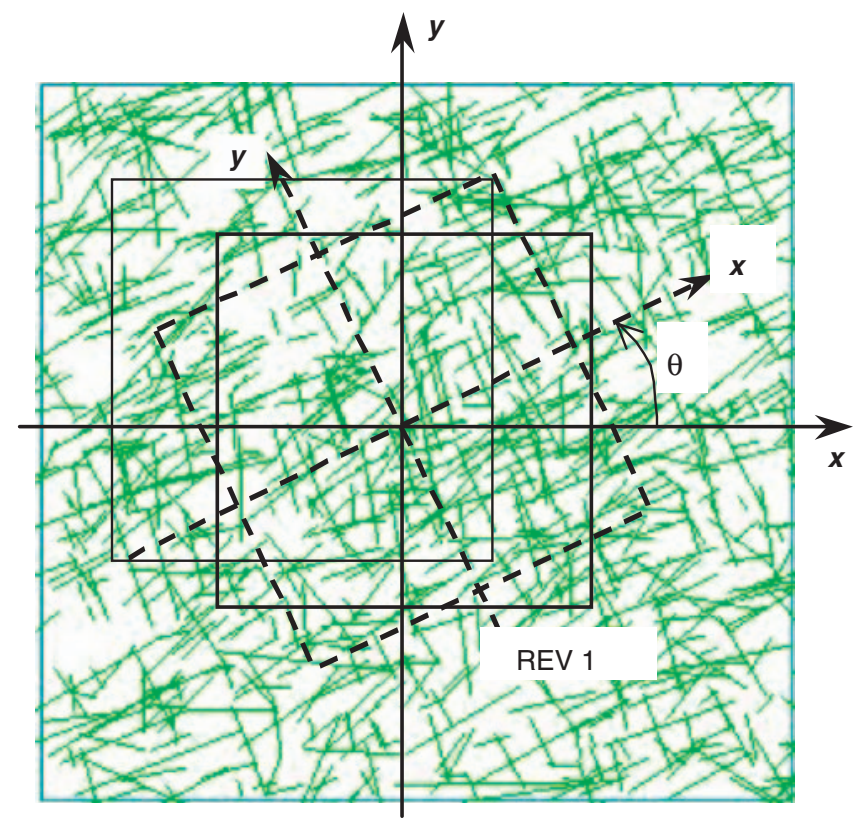

Figure 2. Numerical determination of the elastic parameters in different directions of a heterogeneous medium by rotation of the REV with respect to the medium. 


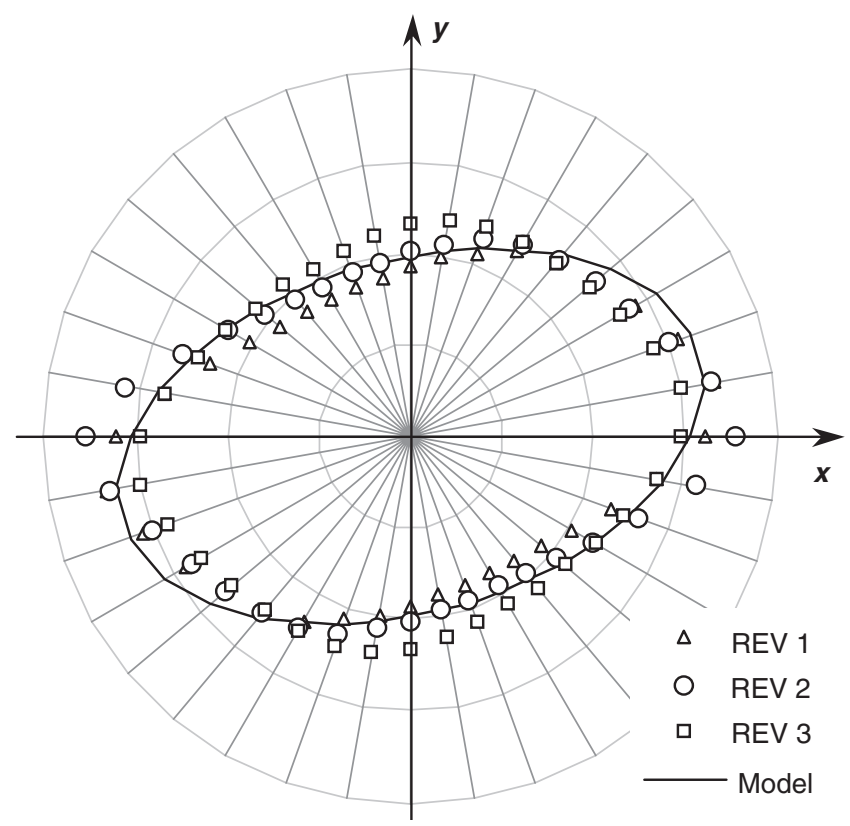

Figure 3. Fitting of the experimental or numerical indicator surfaces obtained for one or several samples by ellipsoids.

the study of the indicator surfaces can constitute a good guideline for this purpose.

Consider that the polar diagrams of $[c(\mathbf{n})]^{-1 / 4}, \sqrt[4]{E(\mathbf{n})},[c(\mathbf{n})]^{-1 / 2}$, or $\sqrt{E(\mathbf{n})}$ have been constructed for one of the several samples (or REVs) by experimental (or numerical) methods. Suppose that they can be well-fitted sufficiently by ellipsoidal surfaces (Figure 3). The problem then is to know how to deduce $\mathbb{C}$ from the fitting parameters. A preliminary question would be to know if any pair of ellipsoids fitted for two different parameters is compatible with the existence of an elasticity tensor. To study these problems, first the classes of materials corresponding to one condition of ellipsoidal indicator surface are characterized, and then the intersection between two different classes is studied.

\section{Material Classes}

The term $\hat{\Phi}_{4}(\mathcal{B})$ is defined as the class of tensors $\mathbb{C}$ for which $\mathrm{F}_{4}(\mathbb{C})$ is an ellipsoid admitting the system $\mathcal{B}$ as principal axes. This class obviously includes $\Phi_{4}(\mathcal{B})$, but, as it will be seen further, is greater than $\Phi_{4}(\mathcal{B})$. In the same way, the classes $\hat{\Phi}_{2}(\mathcal{B}), \hat{\Gamma}_{4}(\mathcal{B}), \hat{\Gamma}_{2}(\mathcal{B}), \hat{\varphi}(\mathcal{B})$, and $\hat{\gamma}(\mathcal{B})$ are defined as the 
classes of tensors $\mathbb{C}$ for which respectively $F_{2}(\mathbb{C}), G_{4}(\mathbb{C}), G_{2}(\mathbb{C}), \mathrm{f}(\mathbb{C})$, and $\mathrm{g}(\mathbb{C})$ is ellipsoidal with principal axes $\mathcal{B}$. The classes $\hat{\Phi}_{4}, \hat{\Phi}_{2}, \hat{\Gamma}_{4}$, and $\hat{\Gamma}_{2}$ are defined as the sets of tensors $\mathbb{C}$ for which the surfaces $F_{4}(\mathbb{C}), F_{2}(\mathbb{C}), G_{4}(\mathbb{C})$, and $G_{2}(\mathbb{C})$ respectively are ellipsoidal:

$$
\hat{\Phi}_{4}=\bigcup_{\mathcal{B}} \hat{\Phi}_{4}(\mathcal{B}), \quad \hat{\Phi}_{2}=\bigcup_{\mathcal{B}} \hat{\Phi}_{2}(\mathcal{B}), \quad \hat{\Gamma}_{4}=\bigcup_{\mathcal{B}} \hat{\Gamma}_{4}(\mathcal{B}), \quad \hat{\Gamma}_{2}=\bigcup_{\mathcal{B}} \hat{\Gamma}_{2}(\mathcal{B})
$$

The spherical classes $\hat{\Phi}_{\mathrm{s}}, \hat{\Gamma}_{\mathrm{s}}, \hat{\varphi}_{\mathrm{s}}$, and $\hat{\gamma}_{\mathrm{s}}$ are defined as the sets of tensors $\mathbb{C}$ for which respectively surfaces $F_{4}(\mathbb{C})\left(\right.$ or $\left.F_{2}(\mathbb{C})\right), G_{4}(\mathbb{C})\left(\right.$ or $G_{2}(\mathbb{C})$ ), $\mathrm{f}(\mathbb{C})$, and $\mathrm{g}(\mathbb{C})$ are spherical. Finally $I_{s}$ represents as the class of isotropic tensors.

At least two different indicator surfaces are required to fully determine $\mathbb{C}$. Hé and Curnier (1995) showed that if the indicator surfaces of parameters $c(\mathbf{n})$ and $h(\mathbf{n})$ defined by Equations (3) and (4) are spherical, then $\mathbb{C}$ is isotropic. This is equivalent to say that if surfaces $F_{4}(\mathbb{C})$ and $\mathrm{f}(\mathbb{C})$ are spherical, then $\mathbb{C}$ is isotropic:

$$
\hat{\Phi}_{s} \cap \hat{\varphi}=I_{s}
$$

Equivalently, if $G_{4}(\mathbb{C})$ and $\mathrm{g}(\mathbb{C})$ are spherical, then $\mathbb{C}$ is isotropic: $\hat{\Gamma}_{\mathrm{s}} \cap \hat{\gamma}_{\mathrm{s}}=I_{s}$.

These results are extended in the following to ellipsoidal surfaces. Two cases are studied: when $F_{4}(\mathbb{C})$ or $F_{2}(\mathbb{C})$ is ellipsoidal with the same planes of symmetry than $\mathrm{f}(\mathbb{C})$, and when $F_{4}(\mathbb{C})$ and $G_{4}(\mathbb{C})$ both are ellipsoidal.

\section{Expression of the Elasticity Tensor for Different Classes}

Let us first determine the explicit expressions of tensors $\mathbb{C}$ or $\mathbb{S}$ for the classes introduced previuosly. For $\mathbb{C} \in \hat{\Phi}_{4}(\mathcal{B})$, the surface $F_{4}(\mathbb{C})$ is an ellipsoid with principal axes $\mathcal{B}$. If $\left(D_{1}, D_{2}, D_{3}\right)$ denote the semidiameters of this ellipsoid, then its equation in the coordinate system $\mathcal{B}$ is:

$$
\frac{x_{1}^{2}}{D_{1}^{2}}+\frac{x_{2}^{2}}{D_{2}^{2}}+\frac{x_{3}^{2}}{D_{3}^{2}}=1
$$

This equation must be equivalent to that given in Table 1 for $\mathrm{F}_{4}(\mathbb{C})$ :

$$
\forall \mathbf{x} ; \quad \frac{x_{1}^{2}}{D_{1}^{2}}+\frac{x_{2}^{2}}{D_{2}^{2}}+\frac{x_{3}^{2}}{D_{3}^{2}}=1 \Leftrightarrow(\mathbf{x} \otimes \mathbf{x}): \mathbb{C}:(\mathbf{x} \otimes \mathbf{x})=1
$$


Using:

$$
\begin{aligned}
(\mathbf{x} \otimes \mathbf{x}): \mathbb{C}:(\mathbf{x} \otimes \mathbf{x})= & c_{11} x_{1}^{4}+c_{22} x_{2}^{4}+c_{33} x_{3}^{4}+\left(2 c_{23}+4 c_{44}\right) x_{2}^{2} x_{3}^{2} \\
& +\left(2 c_{31}+4 c_{55}\right) x_{3}^{2} x_{1}^{2}+\left(2 c_{12}+4 c_{66}\right) x_{1}^{2} x_{2}^{2} \\
& +\left(4 c_{14}+8 c_{56}\right) x_{1}^{2} x_{2} x_{3}+\left(4 c_{25}+8 c_{64}\right) x_{2}^{2} x_{3} x_{1} \\
& +\left(4 c_{36}+8 c_{45}\right) x_{3}^{2} x_{1} x_{2} \\
& +4\left(c_{16} x_{1}^{3} x_{2}+c_{15} x_{1}^{3} x_{3}+c_{26} x_{2}^{3} x_{1}+c_{24} x_{2}^{3} x_{3}\right. \\
& \left.+c_{35} x_{3}^{3} x_{1}+c_{34} x_{3}^{3} x_{2}\right)
\end{aligned}
$$

the equivalence (27) allows one to establish the following relations:

$$
\begin{aligned}
D_{1} & =\left(c_{11}\right)^{-1 / 4}, \quad D_{2}=\left(c_{22}\right)^{-1 / 4}, \quad D_{3}=\left(c_{33}\right)^{-1 / 4} \\
c_{23}+2 c_{44} & =\sqrt{c_{22} c_{33}}, \quad c_{13}+2 c_{55}=\sqrt{c_{11} c_{33}}, \quad c_{12}+2 c_{66}=\sqrt{c_{11} c_{22}} \\
c_{14}+2 c_{56} & =c_{25}+2 c_{46}=c_{36}+2 c_{45}=0 \\
c_{16} & =c_{15}=c_{26}=c_{24}=c_{35}=c_{34}=0
\end{aligned}
$$

An element of $\hat{\Phi}_{4}(\mathcal{B})$ thus depends upon nine intrinsic parameters $\left(c_{11}, c_{22}, c_{33}, c_{12}, c_{23}, c_{13}, c_{14}, c_{25}, c_{36}\right)$ and reads as:

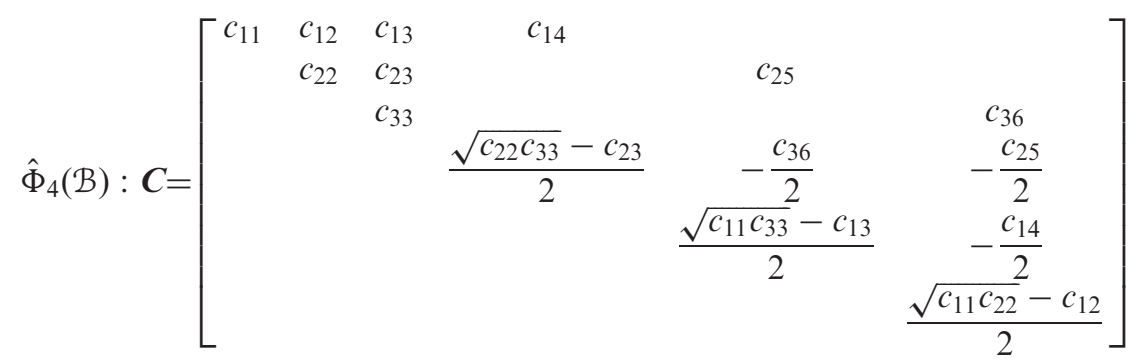

The general expressions of $\boldsymbol{C}$ for the class $\hat{\Phi}_{2}(\mathcal{B})$, as well as the expression of $\underline{\boldsymbol{S}}$ for $\hat{\Gamma}_{4}(\mathcal{B})$ and $\hat{\Gamma}_{2}(\mathcal{B})$ can be established in the same way. For instance, for $\hat{\Phi}_{2}(\mathcal{B}), C$ has the same expression as that of Equation (33), but $c_{44}$, $c_{55}$, and $c_{66}$ are given by Equation (21) instead of Equation (8), and the relation (29) becomes:

$$
D_{1}=\frac{1}{\sqrt{c_{11}}}, \quad D_{2}=\frac{1}{\sqrt{c_{22}}}, \quad D_{3}=\frac{1}{\sqrt{c_{33}}}
$$


$\hat{\Gamma}_{4}(\mathcal{B})$ is found to be defined by the following expression of $\underline{S}$ in which $G_{12}, G_{23}$, and $G_{13}$ are given by (11):

$$
\hat{\Gamma}_{4}(\mathcal{B}): \quad \underline{\mathbf{S}}\left[\begin{array}{cccccc}
\frac{1}{E_{1}} & \frac{-v_{12}}{E_{1}} & \frac{-v_{13}}{E_{1}} & \underline{s}_{14} & & \\
\frac{-v_{12}}{E_{1}} & \frac{1}{E_{2}} & \frac{-v_{23}}{E_{2}} & & \underline{s}_{25} & \\
\frac{-v_{13}}{E_{1}} & \frac{-v_{23}}{E_{2}} & \frac{1}{E_{3}} & & & \underline{S}_{36} \\
& & & \frac{1}{G_{23}} & -\underline{s}_{36} & -\underline{S}_{25} \\
& & & & \frac{1}{G_{13}} & -\underline{s}_{14} \\
& & & & & \frac{1}{G_{12}}
\end{array}\right]
$$

The surfaces $f(\mathbb{C})$ and $g(\mathbb{C})$ are always ellipsoidal. The class $\hat{\varphi}(\mathcal{B})$ (respectively $\hat{\gamma}(\mathcal{B})$ ) represents tensors $\mathbb{C}$ for which ellipsoid $\mathrm{f}(\mathbb{C})$ (respectively $\mathrm{g}(\mathbb{C}))$ has the principal axes $\mathcal{B}$. Let $\mathbb{C} \in \hat{\varphi}(\mathcal{B})$, and note that $\left(d_{1}, d_{2}, d_{3}\right)$ are the semidiameters of $\mathrm{f}(\mathbb{C})$. By comparing Equation (26)-like equation of this ellipsoid to that given in Table 1 for $F_{4}(\mathbb{C})$, and by using the expression:

$$
\begin{aligned}
\boldsymbol{\delta}: \mathbb{C}:(\mathbf{x} \otimes \mathbf{x})= & \left(c_{11}+c_{21}+c_{31}\right) x_{1}^{2}+\left(c_{12}+c_{22}+c_{32}\right) x_{2}^{2}+\left(c_{13}+c_{23}+c_{33}\right) x_{3}^{2} \\
& +2\left(c_{14}+c_{24}+c_{34}\right) x_{2} x_{3}+2\left(c_{15}+c_{25}+c_{35}\right) x_{1} x_{3} \\
& +2\left(c_{16}+c_{26}+c_{36}\right) x_{1} x_{2}
\end{aligned}
$$

one finds:

$$
\begin{aligned}
& d_{1}=\frac{1}{\sqrt{c_{11}+c_{21}+c_{31}}}, \quad d_{2}=\frac{1}{\sqrt{c_{12}+c_{22}+c_{32}}}, \quad d_{3}=\frac{1}{\sqrt{c_{13}+c_{23}+c_{33}}} \\
& c_{14}+c_{24}+c_{34}=c_{15}+c_{25}+c_{35}=c_{16}+c_{26}+c_{36}=0
\end{aligned}
$$

Class $\hat{\gamma}(\mathcal{B})$ is characterized by relations analogous to Equation (38) satisfied by coefficients $\mathrm{s}_{\alpha \beta}$.

Now consider $\mathbb{C} \in \hat{\Phi}_{4}(\mathcal{B})$ defined by Equation (33), and define tensor $\mathbf{H}$ diagonal in $\mathcal{B}$, which is given by:

$$
\mathbf{H}=\operatorname{diag}\left(\sqrt{c_{11}}, \sqrt{c_{22}}, \sqrt{c_{33}}\right)
$$


then one finds:

$$
\forall \mathbf{x} ; \quad(\mathbf{x} \otimes \mathbf{x}): \mathbb{C}:(\mathbf{x} \otimes \mathbf{x})=(\mathbf{x} \cdot \mathbf{H} \cdot \mathbf{x})^{2}
$$

Conversely, if a symmetric matrix $\mathbf{H}$ exists, which allows one to write Equation $(40)$, then $F_{4}(\mathbb{C})$ is obviously ellipsoidal. Therefore one can write:

$$
\hat{\Phi}_{4}=\left\{\mathbb{C} / \exists \mathbf{H} ; \forall \mathbf{x} ; \quad(\mathbf{x} \otimes \mathbf{x}): \mathbb{C}:(\mathbf{x} \otimes \mathbf{x})=(\mathbf{x} . \mathbf{H} \cdot \mathbf{x})^{2}\right\}
$$

In the same way:

$$
\begin{array}{ll}
\hat{\Phi}_{2}=\{\mathbb{C} / \exists \mathbf{H} ; \forall \mathbf{x} ; & (\mathbf{x} \otimes \mathbf{x}): \mathbb{C}:(\mathbf{x} \otimes \mathbf{x})=(\mathbf{x} . \mathbf{H} \cdot \mathbf{x})(\mathbf{x} \cdot \mathbf{x})\} \\
\hat{\Gamma}_{4}=\{\mathbb{C} / \exists \mathbf{H} ; \forall \mathbf{x} ; & \left.(\mathbf{x} \otimes \mathbf{x}): \mathbb{S}:(\mathbf{x} \otimes \mathbf{x})=(\mathbf{x} . \mathbf{H} \cdot \mathbf{x})^{2}\right\} \\
\hat{\Gamma}_{2}=\{\mathbb{C} / \exists \mathbf{H} ; \forall \mathbf{x} ; & (\mathbf{x} \otimes \mathbf{x}): \mathbb{S}:(\mathbf{x} \otimes \mathbf{x})=(\mathbf{x} . \mathbf{H} . \mathbf{x})(\mathbf{x} \cdot \mathbf{x})\}
\end{array}
$$

It is interesting to note that the materials of classes $\hat{\Phi}_{4}, \hat{\Phi}_{2}, \hat{\Gamma}_{4}$, and $\hat{\Gamma}_{2}$ in general are not orthotropic, and cannot even have any plane of symmetry. As a matter of fact, a plane of symmetry of $\mathbb{C}$ is necessarily a plane of symmetry of its indicator surfaces. If the three semi-diameters of the ellipsoid $\mathrm{F}_{4}(\mathbb{C})$, for instance, are different then its only planes of symmetry are those of system $\mathcal{B}$, which manifestly do not constitute planes of symmetry for $\boldsymbol{C}$ given in Equation (33). In consequence, the method of characterization of the material's anisotropy based on the analysis of the number and orientation of its planes of reflective symmetry (Cowin and Mehrabadi, 1987, 1995) would not give significant result for these classes of materials.

\section{MATERIAL IDENTIFICATION}

In this section let us study some cases in which two ellipsoidal surfaces allows us to fully determine $\mathbb{C}$.

\section{Intersection of $\hat{\Phi}_{4}(\mathcal{B})$ and $\hat{\varphi}(\mathcal{B})$}

Suppose that $F_{4}(\mathbb{C})$ is ellipsoidal. Since $\mathrm{f}(\mathbb{C})$ is always ellipsoidal, the interesting case to consider is when the two ellipsoids have the same principal axes, i.e., when $\mathbb{C} \in \hat{\Phi}_{4}(\mathcal{B}) \cap \hat{\varphi}(\mathcal{B})$. Then $\boldsymbol{C}$ is given by Equation (33), and Equation (38) on $\hat{\varphi}(\mathcal{B})$ implies that $c_{14}=c_{25}=c_{36}=0$; so $\mathbb{C}$ is orthotropic. The following result is obtained in this way:

$$
\hat{\Phi}_{4}(\mathcal{B}) \cap \hat{\varphi}(\mathcal{B})=\Phi_{4}(\mathcal{B})
$$


In the same way, it can be shown that:

$$
\hat{\Phi}_{2}(\mathcal{B}) \cap \hat{\varphi}(\mathcal{B})=\Phi_{2}(\mathcal{B})
$$

The same data $c(\mathbf{n})$ can be utilized for defining two surfaces $F_{2}(\mathbb{C})$ and $F_{4}(\mathbb{C})$. According to that data a best ellipsoidal fitting is obtained for $F_{4}(\mathbb{C})$ or for $F_{2}(\mathbb{C})$, an element of $\hat{\Phi}_{4}$ or of $\hat{\Phi}_{2}$ is found. Equations (45) and (46) mean that, when fitting $F_{4}(\mathbb{C})$ or $F_{2}(\mathbb{C})$ by an ellipsoid, if the principal axes of the ellipsoid are constrained to have the same direction as that for $f(\mathbb{C})$, then an orthotropic material is found. The parameters of $\mathbb{C}$ can be, in these cases, fully determined from the semidiameters of the two ellipsoids by using Equations (29), (30), and (37) in the first case and Equations (21), (34), and (37) in the second case.

Now consider the case of materials for which $c(\mathbf{n})$ is constant. In this case $\mathbb{C} \in \hat{\Phi}_{\mathrm{s}}$. Since for every system of axes $\mathcal{B}, \hat{\Phi}_{\mathrm{s}} \subset \hat{\Phi}_{4}(\mathcal{B})$, one can deduce from Equation (45) that $\hat{\Phi}_{\mathrm{S}} \cap \hat{\varphi}(\mathcal{B}) \subset \hat{\Phi}_{4}(\mathcal{B})$, and can then establish:

$$
\hat{\Phi}_{\mathrm{S}} \cap \hat{\varphi}(\mathcal{B})=\Phi_{\mathrm{S}}(\mathcal{B})
$$

An element of $\Phi_{\mathrm{s}}(\mathcal{B})$ is given by Equation (33) in which $c_{14}=c_{25}=c_{36}=0$ and $c_{11}=c_{22}=c_{33}$ If, in addition, $\mathrm{f}(\mathbb{C})$ is spherical, then by writing $d_{1}=d_{2}=d_{3}$ in Equation (37) for an element of $\Phi_{\mathrm{s}}(\mathcal{B})$, one finds that $\mathbb{C}$ is isotropic. Equation (47) reduces in this case to the result given by Hé and Curnier (1995):

$$
\hat{\Phi}_{\mathrm{s}} \cap \hat{\varphi}_{\mathrm{s}}=I_{S}
$$

Equivalent relations to Equations (45)-(47) can be written for $\hat{\Gamma}$-type materials, for instance, for the first relation, $\hat{\Gamma}_{4}(\mathcal{B}) \cap \hat{\gamma}(\mathcal{B})=\hat{\Gamma}_{4}(\mathcal{B})$.

It is interesting to note that Equation (47) implies that the elements of $\hat{\Phi}_{\mathrm{s}}$ (and equivalently $\hat{\Gamma}_{\mathrm{s}}$ ) are all orthotropic. As a matter of fact, for every $\mathbb{C} \in \hat{\Phi}_{\mathrm{s}}$, if $\mathcal{B}$ denotes a system of principal axes of $\mathrm{f}(\mathbb{C})$, then $\mathbb{C} \in \hat{\varphi}(\mathcal{B})$, and then according to Equation (47), $\mathbb{C} \in \Phi_{\mathrm{s}}(\mathcal{B})$ and hence $\mathbb{C}$ is orthotropic:

If the elastic coefficient $c(\mathbf{n})$ (or Young's modulus $E(\mathbf{n}))$ is constant in all directions, then the material is orthotropic.

\section{Intersection of $\hat{\Phi}_{4}$ and $\hat{\Gamma}_{4}$}

Consider in this section the case of materials for which both indicator surfaces $F_{4}(\mathbb{C})$ and $G_{4}(\mathbb{C})$ are ellipsoidal. First consider the case in which these ellipsoids have a common set of principal axes $\mathcal{B}$. Then the more general case is considered in which this condition is not supposed a priori. 
INTERSECTION OF $\hat{\Phi}_{4}(\mathcal{B}) A N D \hat{\Gamma}_{4}(\mathcal{B})$

If $\mathbb{C} \in \hat{\Phi}_{4}(\mathcal{B}) \cap \hat{\Gamma}_{4}(\mathcal{B})$, then the planes of the coordinate system $\mathcal{B}$ constitute planes of reflexive symmetry for the surfaces $F_{4}(\mathbb{C})$ and $G_{4}(\mathbb{C})$, or also for the scalar functions $(\mathbf{n} \otimes \mathbf{n}): \mathbb{C}:(\mathbf{n} \otimes \mathbf{n})$ and $(\mathbf{n} \otimes \mathbf{n}): \mathbb{S}:(\mathbf{n} \otimes \mathbf{n})$. This property for the function $(\mathbf{n} \otimes \mathbf{n}): \mathbb{C}:(\mathbf{n} \otimes \mathbf{n})$ is equivalent to Equations (30) and (31) and also to the expression of the matrix $\boldsymbol{C}$ given by Equations (49) and (50). The same property for $(\mathbf{n} \otimes \mathbf{n}): \mathbb{S}:(\mathbf{n} \otimes \mathbf{n})$ is equivalent to the $\underline{\boldsymbol{S}}$ given by Equations (49) and (51):

$$
\boldsymbol{C}=\left[\begin{array}{cc}
\mathbf{M} & \mathbf{D} \\
\mathbf{D} & \frac{1}{2} \mathbf{N}
\end{array}\right], \quad \underline{\boldsymbol{S}}=\left[\begin{array}{cc}
\mathbf{M}^{\prime} & 2 \mathbf{D}^{\prime} \\
2 \mathbf{D}^{\prime} & 2 \mathbf{N}^{\prime}
\end{array}\right]
$$

with:

$$
\begin{gathered}
\mathbf{M}=\left[\begin{array}{lll}
c_{11} & c_{12} & c_{13} \\
& c_{22} & c_{23} \\
& & c_{33}
\end{array}\right], \quad \mathbf{N}=\left[\begin{array}{ccc}
2 c_{44} & -c_{36} & -c_{25} \\
& 2 c_{55} & -c_{14} \\
& & 2 c_{66}
\end{array}\right], \quad \mathbf{D}=\left[\begin{array}{lll}
c_{14} & & \\
& c_{25} & \\
& & c_{36}
\end{array}\right] \\
\mathbf{M}^{\prime}=\left[\begin{array}{lll}
\underline{S}_{11} & \underline{S}_{12} & \underline{S}_{13} \\
& \underline{\underline{S}}_{22} & \underline{\underline{S}}_{23} \\
& & \underline{S}_{33}
\end{array}\right], \quad \mathbf{N}^{\prime}=\frac{1}{2}\left[\begin{array}{ccc}
\underline{S}_{44} & -\underline{s}_{36} & -\underline{s}_{25} \\
& \underline{\underline{S}}_{55} & -\underline{s}_{14} \\
& & \underline{S}_{66}
\end{array}\right], \quad \mathbf{D}^{\prime}=\frac{1}{2}\left[\begin{array}{lll}
\underline{S}_{14} & & \\
& \underline{S}_{25} & \\
& & \underline{S}_{36}
\end{array}\right]
\end{gathered}
$$

Equation $\mathbb{C}: \mathbb{S}=\mathbb{\rrbracket}$, or $c_{\alpha \beta} s_{\beta \gamma}=\delta_{\alpha \beta}$, implies the following matrix equations in which $\mathbf{I}$ represents the $3 \times 3$ unit matrix:

$$
\begin{aligned}
\mathbf{M} \mathbf{M}^{\prime}+2 \mathbf{D D ^ { \prime }} & =\mathbf{I} \\
\mathbf{N} \mathbf{N}^{\prime}+2 \mathbf{D D ^ { \prime }} & =\mathbf{I} \\
\mathbf{M D}^{\prime}+\mathbf{D N}^{\prime} & =0 \\
\mathbf{D} \mathbf{M}^{\prime}+\mathbf{N D}^{\prime} & =0
\end{aligned}
$$

Symmetry and positive definite properties of $\mathbb{C}$ and $\mathbb{S}$ imply that $\mathbf{M}, \mathbf{M}^{\prime}$, $\mathbf{N}$, and $\mathbf{N}^{\prime}$ are symmetric and positive definite.

In addition to these relations, Equations (8) and (10) must be satisfied for $F_{4}(\mathbb{C})$ and $G_{4}(\mathbb{C})$ to be ellipsoidal. 
Equations (50)-(55) which express the symmetry of $(\mathbf{n} \otimes \mathbf{n}): \mathbb{C}:(\mathbf{n} \otimes \mathbf{n})$ and $(\mathbf{n} \otimes \mathbf{n}): \mathbb{S}:(\mathbf{n} \otimes \mathbf{n})$ with respect to the planes of the coordinate system have been studied in Appendix 2. It has been shown that they can have three different types of solutions and only one of these types shown here, is compatible with which (8) and (10).

The first type (Case 1) is characterized by a parameter $\eta_{1} \neq 0$ and by, in particular, the following relations between the parameters (Equations (10) and (11) in Appendix 2):

$$
\begin{aligned}
& \underline{s}_{44}=\left(1-2 \eta_{1}\right) / 2 c_{44}, \underline{s}_{22}=c_{33} /\left[c_{22} c_{33}-\left(c_{23}\right)^{2}\right], \\
& \underline{s}_{33}=c_{22} /\left[c_{22} c_{33}-\left(c_{23}\right)^{2}\right], \underline{s}_{23}=-c_{23} /\left[c_{22} c_{33}-\left(c_{23}\right)^{2}\right]
\end{aligned}
$$

Substituting these relations in the first Equation of (10), one finds:

$$
\frac{1-2 \eta_{1}}{2 c_{44}}=\frac{\sqrt{c_{22} c_{33}}}{c_{22} c_{33}-c_{23}^{2}}+\frac{c_{23}}{c_{22} c_{33}-c_{23}^{2}}=\frac{1}{\sqrt{c_{22} c_{33}}-c_{23}}
$$

Since this equation, compared to Equation (8) implies $\eta_{1}=0$, Case 1 is incompatible with Equations (8) and (10).

The second type of solution (Case 2.1) is characterized by a parameter $\eta<0$ and a parameter $\kappa$ satisfying:

$$
(1-4 \eta)^{2} \kappa^{2}+(2-9 \eta) \eta \kappa+\eta^{2}=0
$$

In this case the following relation between the parameters can be obtained (deduced from Equations (34) and (36) in Appendix 2):

$$
2 c_{44}+c_{33}=\beta^{2}(1-k) \sqrt{c_{22} c_{33}}
$$

where $\beta=-\eta /[\kappa(1-4 \eta)]$. This equation, compared to Equation (8) leads to $\beta^{2}(1-\kappa)=1$ and then to $\eta^{2}(1-\kappa) /[\kappa(1-4 \eta)]^{2}=1$. Substituting in this equation for $\kappa^{2}(1-4 \eta)^{2}$ by Equation $(56)$, one finds $\eta[\kappa(1-5 \eta)+\eta]=0$. Since $\eta=0$ is not compatible with Case 2.1, it is deduced that $\kappa=-\eta /(1-5 \eta)$. By substituting this expression of $\kappa$ in Equation (56) one finds $\eta^{3}(1-4 \eta)=0$, which is also incompatible with the condition $\eta<0$ characterizing Case 2.1. Thus, this case is also incompatible with Equation (8).

Therefore, the only type of solution of Equations (50)-(55) compatible with Equations (8) and (9) is the last type described in Appendix 2 (Case 2.2) in which $\mathbf{D}=\mathbf{D}^{\prime}=\mathbf{0}$. 
In conclusion, for $\mathbb{C} \in \hat{\Phi}_{4}(\mathcal{B}) \cap \hat{\Gamma}_{4}(\mathcal{B})$, the matrices $\boldsymbol{C}$ and $\underline{\boldsymbol{S}}$ are given in the coordinate system $\mathcal{B}$ by Equation (49) with $\mathbf{D}=\mathbf{D}^{\prime}=\mathbf{0}$. This means that $\mathbb{C} \in \hat{\Phi}_{4}(\mathcal{B}) \cap \hat{\Gamma}_{4}(\mathcal{B})$ is orthotropic and that its planes of orthotropy are given by $B$. This is a main result of the present article. It allows one to write:

$$
\hat{\Phi}_{4}(\mathcal{B}) \cap \hat{\Gamma}_{4}(\mathcal{B})=\hat{\Phi}_{4}(\mathcal{B}) \cap \hat{\Gamma}_{4}(\mathcal{B})
$$

and, by using Equation (12):

$$
\hat{\Phi}_{4}(\mathcal{B}) \cap \hat{\Gamma}_{4}(\mathcal{B})=\Psi(\mathcal{B})
$$

In this case, matrices $\boldsymbol{C}$ and $\underline{\boldsymbol{S}}$ respectively are given by Equations (13) and (14). The parameters $c_{11}, c_{22}$, and $c_{33}$ can be deduced from the semidiameters of $F_{4}(\mathbb{C})$ (Equations $(29)$ ) and the semidiameters $\left\{\sqrt[4]{E_{1}}, \sqrt[4]{E_{2}}, \sqrt[4]{E_{3}}\right\}$ of $G_{4}(\mathbb{C})$, and $\eta$, and $v$ are deduced from Equation (15). The tensor $\mathbb{C}$ in this way is fully determined.

INTERSECTIONS OF $\hat{\Phi}_{S} A N D \hat{\Gamma}_{S}$

First $\hat{\Phi}_{\mathrm{S}} \cap \hat{\Gamma}_{4}(\mathcal{B})$ is studied. Since $\hat{\Phi}_{\mathrm{s}} \subset \hat{\Phi}_{4}(\mathcal{B})$, Equation (58) implies that $\hat{\Phi}_{S} \cap \hat{\Gamma}_{4}(\mathcal{B}) \subset \Psi(\mathcal{B})$. Then if $\mathbb{C} \in \hat{\Phi}_{S} \cap \hat{\Gamma}_{4}(\mathcal{B}), C$ is given by Equation (13) and the relations (29) lead to $c_{11}=c_{22}=c_{33}$. This means that $\mathbb{C}$ is isotropic. It can be deduced that $\hat{\Phi}_{s} \mathbb{C} \hat{\Gamma}_{4}(\mathcal{B}) \subset I_{s}$ and then shown that:

$$
\hat{\Phi}_{\mathrm{S}} \cap \hat{\Gamma}_{4}(\mathcal{B})=I_{S}
$$

By using Equation (24) of $\hat{\Gamma}_{4}$, the equality Equation (59) can be extended to:

$$
\hat{\Phi}_{\mathrm{S}} \cap \hat{\Gamma}_{4}=I_{S}
$$

Since $\hat{\Gamma}_{s} \subset \hat{\Gamma}_{4}$, it can be deduced from Equation (60) that $\hat{\Phi}_{s} \cap \hat{\Gamma}_{s} \subset I_{s}$ and since conversely $I_{s} \subset \hat{\Phi}_{\mathrm{s}}$ and $I_{s} \subset \hat{\Gamma}_{s}$, then:

$$
\hat{\Phi}_{\mathrm{s}} \cap \hat{\Gamma}_{s}=I_{s}
$$

This result means that: if $E(\mathbf{n})$ and $c(\mathbf{n})$ are both constant in all directions, $\mathbb{C}$ is isotropic.

The coefficients $E$ and $v$ corresponding to this isotropic elasticity tensor can be deduced from the radii of the spheres $F_{4}(\mathbb{C})$ and $G_{4}(\mathbb{C})$, noted respectively $R$ and $\rho$, by:

$$
\rho^{4}=E, \quad R^{4}=\frac{(1+v)(1-2 v)}{(1-v) E}
$$


INTERSECTION OF $\hat{\Phi}_{4} A N D \hat{\Gamma}_{4}$

Now suppose that $\mathbb{C} \in \hat{\Phi}_{4}(\mathcal{B}) \cap \hat{\Gamma}_{4}$. In this case, $\mathcal{B}$ defines a system of principal axes for ellipsoid $F_{4}(\mathbb{C})$, but, a priori, not necessarily for $G_{4}(\mathbb{C})$. Since $\mathbb{C} \in \hat{\Phi}_{4}(\mathcal{B})$, matrix $\boldsymbol{C}$ has Equation (33) in the coordinate system $\mathcal{B}$. Consider now tensor $\mathbf{Q}$, diagonal in the basis $\mathcal{B}$, and given by:

$$
\mathbf{Q}=\operatorname{diag}\left(\frac{1}{\sqrt[4]{c_{11}}}, \frac{1}{\sqrt[4]{c_{22}}}, \frac{1}{\sqrt[4]{c_{33}}}\right)
$$

Let tensor $\mathbb{C}^{\prime}$ be deduced from $\mathbb{C}$ by the following transformation (Pouya, 2000; Pouya and Zaoui, 2005):

$$
C_{i j k l}^{\prime}=Q_{i m} Q_{j n} Q_{k p} Q_{l q} C_{m n p q}
$$

It can be verified that $\mathbb{C}^{\prime}$ has the symmetries of an elasticity tensor and is positive definite. So it represents a new elasticity tensor. The matrix $\boldsymbol{C}^{\prime}$ has Equation (33) in the coordinate system $\mathcal{B}$ with, in particular:

$$
\begin{aligned}
& c_{11}^{\prime}=1, \quad c_{12}^{\prime}=\frac{c_{12}}{\sqrt{c_{11} c_{22}}}, \quad c_{14}^{\prime}=\frac{c_{14}}{\sqrt[4]{c_{11}^{2} c_{22} c_{33}}}, \\
& c_{44}^{\prime}=\frac{c_{44}}{\sqrt{c_{22} c_{33}}}, \quad c_{45}^{\prime}=\frac{c_{45}}{\sqrt{c_{11}^{2} c_{22} c_{33}}}
\end{aligned}
$$

The other elements of $C^{\prime}$ are deduced from Equation (65) by index permutation. Equations (30) and (31) are well satisfied; for instance:

$$
c_{44}^{\prime}=\frac{\sqrt{c_{22}^{\prime} c_{33}^{\prime}}-c_{23}^{\prime}}{2}, \quad c_{45}^{\prime}=-\frac{c_{36}^{\prime}}{2}
$$

Since $c_{11}^{\prime}=c_{22}^{\prime}=c_{33}^{\prime}=1$, then $\mathbb{C}^{\prime} \in \hat{\Phi}_{\mathrm{s}}$. Denoting $\mathbb{S}^{\prime}=\mathbb{C}^{-1}$ and $\mathbf{P}=\mathbf{Q}^{-1}$, it can be deduced from Equation (64) that:

$$
S_{i j k l}^{\prime}=P_{i m} P_{j n} P_{k p} P_{l q} S_{m n p q}
$$

Since it is assumed that $\mathbb{C} \in \hat{\Gamma}_{4}$, according to Equation (43), a reversible tensor $\mathbf{T}$ exists which allows one to write:

$$
\forall \mathbf{x} ; \quad(\mathbf{x} \otimes \mathbf{x}): \mathbb{S}:(\mathbf{x} \otimes \mathbf{x})=(\mathbf{x} \cdot \mathbf{T} \cdot \mathbf{x})^{2}
$$


Equation (66) then implies that:

$$
\forall \mathbf{x} ; \quad(\mathbf{x} \otimes \mathbf{x}): \mathbb{S}^{\prime}:(\mathbf{x} \otimes \mathbf{x})=\left(\mathbf{x} \cdot \mathbf{T}^{\prime} \cdot \mathbf{x}\right)^{2}
$$

with:

$$
\mathbf{T}^{\prime}=\mathbf{P T P}
$$

According to Equation (43), this means that $\mathbb{C}^{\prime} \in \hat{\Gamma}_{4}$. Since already $\mathbb{C}^{\prime} \in \hat{\Phi}_{\mathrm{s}}$, Equation (60) implies that $\mathbb{C}^{\prime} \in I_{S}$. Then writing Equation (64) as:

$$
C_{i j k l}=P_{i m} P_{j n} P_{k p} P_{l q} C_{m n p q}^{\prime}
$$

and comparing with Equation (16), it can be deduced that $\mathbb{C} \in \Psi(\mathcal{B})$. This implies that $\hat{\Phi}_{4}(\mathcal{B}) \cap \hat{\Gamma}_{4} \subseteq \Psi_{4}(\mathcal{B})$. Since conversely $\Psi(B) \subset \hat{\Phi}_{4}(\mathcal{B})$ and $\Psi(\mathcal{B}) \subset \hat{\Gamma}_{4}$, one obtains:

$$
\hat{\Phi}_{4}(\mathcal{B}) \cap \hat{\Gamma}_{4}=\Psi(\mathcal{B})
$$

By using Equation (19) and (24) of $\Psi$ and $\hat{\Phi}_{4}$, the result i.e., Equation (69) allows one to write:

$$
\hat{\Phi}_{4} \cap \hat{\Gamma}_{4}\left(\bigcup_{\mathcal{B}} \hat{\Phi}_{4}(\mathcal{B})\right) \cap \hat{\Gamma}_{4}=\bigcup_{\mathcal{B}}\left(\hat{\Phi}_{4}(\mathcal{B}) \cap \hat{\Gamma}_{4}\right)=\bigcup_{\mathcal{B}} \Psi(\mathcal{B})=\Psi
$$

and finally:

$$
\hat{\Phi}_{4} \cap \hat{\Gamma}_{4}=\Psi
$$

This equation means that, if the surfaces $F_{4}(\mathbb{C})$ and $G_{4}(\mathbb{C})$ are both ellipsoidal, then they have necessarily the same (or a common system of) principal axes. Moreover, the material is orthotropic and the common system of principal axes of the two ellipsoids defines also the planes of orthotropy.

In consequence, in the phenomenological modeling of a materials elasticity, when the values of $E(\mathbf{n})$ and $c(\mathbf{n})$ are given and one searches for an ellipsoidal approximation of both surfaces $F_{4}(\mathbb{C})$ and $G_{4}(\mathbb{C})$, then the two ellipsoids fitting these surfaces must be constrained to have the same 
directions of principal axes. Only under this condition can all the parameters of $\mathbb{C}$, which will be defined in this case by Equations (13) or (14), be determined. They are deduced from the semidiameters of the two ellipsoids and Equation (15).

\section{DISCUSSION AND CONCLUSIONS}

In this article, some cases of ellipsoidal anisotropy and the method and conditions of elasticity tensor determination from ellipsoidal fitting parameters were studied.

As mentioned previously, the solution of many basic problems of elasticity for $\Psi$-type materials can be deduced by a linear transformation from the solutions known for isotropic materials (Pouya, 2000; Pouya and Zaoui, 2005). It should be emphasized that some problems which can be solved in this way for $\Psi$-type materials, such as the Green function of a halfspace (Pouya and Zaoui, 2005), cannot be approached by other methods such as the Stroh formalism (Stroh, 1958; Ting, 1996) since they do not present any plane of symmetry. The author's ongoing investigations show that some of these closed-form solutions, for instance the Green function solution for infinite space, can be established for the family $\hat{\Phi}_{4}$. This increases the interest of this type of anisotropy. Class $\hat{\Phi}_{2}$ recovers, as mentioned above, a large family of models already used for damaged or heterogeneous materials, which are based on a second-order damage tensor (Kachanov, 1992). Ellipsoidal models could also be used for the approximation of more complex damage models based on fourth-order tensors (Zheng, 1997). It can be noticed that in spite of more interesting properties of $\hat{\Phi}_{4}$ and $\hat{\Phi}_{2}$, classes $\hat{\Gamma}_{4}$ and $\hat{\Gamma}_{2}$, have drawn more attention in the literature. For instance, $\Gamma_{4}$ and $\Gamma_{2}$ are the only families mentioned by Lekhnitskii (1963) when quoting Saint Venant's work.

In conclusion, the concept of ellipsoidal anisotropy, when it fits the materials data well, seems an attractive guideline for phenomenological modeling of the amorphous or damaged materials elasticity. As a way to define anisotropic models with a reduced number of parameters, it constitutes an alternative to the method based on the number and orientation of planes of reflective symmetry (Cowin and Mehrabadi, 1987, 1995). The two methods recover different families of materials. Besides providing potential tools for modeling the materials anisotropy, ellipsoidal anisotropy classes, which are an extension of the families introduced by Saint Venant, present interesting theoretical features concerning the resolution of elastic body problems. 


\section{APPENDIX 1}

\section{The Indicator Surface of the Young's Modulus}

The indicator surface of the Young's modulus is defined by the equation (see the main text):

$$
[(\mathbf{x} \otimes \mathbf{x}): S:(\mathbf{x} \otimes \mathbf{x})]^{2}=(\mathbf{x} \cdot \mathbf{x})^{3}
$$

Suppose that this surface is an ellipsoid, the equation of an ellipsoid can be written as:

$$
\mathbf{x} \cdot \mathbf{H} \cdot \mathbf{x}=1
$$

where $\mathbf{H}$ is a second-rank symmetric and positive definite tensor. Then, one can write the following equivalence:

$$
\forall \mathbf{x} ; \quad[(\mathbf{x} \otimes \mathbf{x}): \mathbb{S}:(\mathbf{x} \otimes \mathbf{x})]^{2}=(\mathbf{x} \cdot \mathbf{x})^{3} \Leftrightarrow \mathbf{x} \cdot \mathbf{H} \cdot \mathbf{x}=1
$$

For every vector $\mathbf{x}$, if $\rho=(\mathbf{x} \cdot \mathbf{H} \cdot \mathbf{x})^{1 / 2}$, and $\mathbf{x}^{\prime}=\mathbf{x} / \rho$, one finds:

$$
\mathbf{x}^{\prime} \cdot \mathbf{H} \cdot \mathbf{x}^{\prime}=1
$$

Then, according to Equation (73), we have $\left[\left(\mathbf{x}^{\prime} \otimes \mathbf{x}^{\prime}\right): \mathbb{S}:\left(\mathbf{x}^{\prime} \otimes \mathbf{x}^{\prime}\right)\right]^{2}=\left(\mathbf{x}^{\prime} \cdot \mathbf{x}^{\prime}\right)^{3}$, and by taking into account Equation (74), $\left[\left(\mathbf{x}^{\prime} \otimes \mathbf{x}^{\prime}\right): \mathbb{S}:\left(\mathbf{x}^{\prime} \otimes \mathbf{x}^{\prime}\right)\right]^{2}=$ $\left(\mathbf{x}^{\prime} \cdot \mathbf{x}^{\prime}\right)^{3}\left(\mathbf{x}^{\prime} \cdot \mathbf{H} \cdot \mathbf{x}^{\prime}\right)$. Multiplying the two sides of this equality by $\rho^{8}$, one finds: $[(\mathbf{x} \otimes \mathbf{x}): \mathbb{S}:(\mathbf{x} \otimes \mathbf{x})]^{2}=(\mathbf{x} \cdot \mathbf{x})^{3}(\mathbf{x} \cdot \mathbf{H} \cdot \mathbf{x})$. Therefore,

$$
\forall \mathbf{x} ; \quad[(\mathbf{x} \otimes \mathbf{x}): S:(\mathbf{x} \otimes \mathbf{x})]^{2}=(\mathbf{x} \cdot \mathbf{x})^{3}(\mathbf{x} \cdot \mathbf{H} \cdot \mathbf{x})
$$

If $\mathbf{x}=(x, 1,0)$, the expression $(\mathbf{x} \otimes \mathbf{x}): \mathbb{S}:(\mathbf{x} \otimes \mathbf{x})$ becomes a fourth-order polynomial in the scalar variable $x$. This polynomial has no real root, because if $(\mathbf{x} \otimes \mathbf{x}): \mathbb{S}:(\mathbf{x} \otimes \mathbf{x})=0$, owing to positive definite assumption for $\mathbb{S}$, one must have $\mathbf{x} \otimes \mathbf{x}=0$, and then $\mathbf{x}=0$, and this is incompatible with $\mathbf{x}=(x, 1,0)$. Therefore, this fourth-order polynomial in $x$ can be decomposed in the product of two irreducible second-order polynomials:

$$
(\mathbf{x} \otimes \mathbf{x}): \mathbb{S}:(\mathbf{x} \otimes \mathbf{x})=P_{1}(x) P_{2}(x)
$$

Equation (75) implies then:

$$
\left[P_{1}(x)\right]^{2}\left[P_{2}(x)\right]^{2}=\left(x^{2}+1\right)^{3}\left(H_{11} x^{2}+2 H_{12} x+H_{22}\right)
$$


Since all polynomials in the two sides of this equality are irreducible, $P_{1}(x)$ and $P_{2}(x)$ must be proportional to $x^{2}+1$ or $H_{11} x^{2}+2 H_{12} x+H_{22}$. Then, since the polynomials appear in the left side at pair power and, in the right, at odd power, one can establish that Equation (77) is possible only if $H_{11} x^{2}+2 H_{12} x+H_{22}$ is proportional to $x^{2}+1$. This means that $H_{11}=H_{22}$ and $H_{12}=0$. In the same way one can establish that $H_{11}=H_{33}, H_{13}=0$, and $H_{23}=0$, and globally that $\mathbf{H}$ is proportional to the unit tensor. Therefore, the surface defined by Equation (72) is a sphere.

The indicating surface of Young's modulus thus cannot be an ellipsoid different from a sphere.

\section{APPENDIX 2}

\section{Conditions of Three Orthogonal Planes of Symmetry for $c(n)$ and $E(n)$}

The conditions of symmetry of the scalar functions $(\mathbf{n} \otimes \mathbf{n}): \mathbb{C}:(\mathbf{n} \otimes \mathbf{n})$ and $(\mathbf{n} \otimes \mathbf{n}): \mathbf{S}:(\mathbf{n} \otimes \mathbf{n})$ with respect to the planes of the coordinate system have been considered in the main text. They are equivalent to suppose that the following matrices:

$$
\begin{aligned}
& \mathbf{A}=\left[\begin{array}{lll}
a_{1} & c_{3} & c_{2} \\
c_{3} & a_{2} & c_{1} \\
c_{2} & c_{1} & a_{3}
\end{array}\right], \quad \mathbf{B}=\left[\begin{array}{ccc}
b_{1} & -d_{3} & -d_{2} \\
-d_{3} & b_{2} & -d_{1} \\
-d_{2} & -d_{1} & b_{3}
\end{array}\right], \quad \mathbf{D}=\left[\begin{array}{lll}
d_{1} & & \\
& d_{2} & \\
& & d_{3}
\end{array}\right] \\
& \mathbf{A}^{\prime}=\left[\begin{array}{lll}
a_{1}^{\prime} & c_{3}^{\prime} & c_{2}^{\prime} \\
c_{3}^{\prime} & a_{2}^{\prime} & c_{1}^{\prime} \\
c_{2}^{\prime} & c_{1}^{\prime} & a_{3}^{\prime}
\end{array}\right], \quad \mathbf{B}^{\prime}=\left[\begin{array}{ccc}
b_{1}^{\prime} & -d_{3}^{\prime} & -d_{2}^{\prime} \\
-d_{3}^{\prime} & b_{2}^{\prime} & -d_{1}^{\prime} \\
-d_{2}^{\prime \prime} & -d_{1}^{\prime} & b_{3}^{\prime}
\end{array}\right], \quad \mathbf{D}^{\prime}=\left[\begin{array}{lll}
d_{1}^{\prime} & & \\
& d_{2}^{\prime} & \\
& & d_{3}^{\prime}
\end{array}\right]
\end{aligned}
$$

with $\mathbf{A}, \mathbf{A}^{\prime}, \mathbf{B}$, and $\mathbf{B}^{\prime}$ being positive-definite, satisfy the following equations:

$$
\begin{aligned}
\mathbf{A} \mathbf{A}^{\prime} & =I-2 \mathbf{D D}^{\prime} \\
\mathbf{B B}^{\prime} & =I-2 \mathbf{D D}^{\prime} \\
\mathbf{A D}^{\prime}+\mathbf{D B}^{\prime} & =0 \\
\mathbf{D} \mathbf{A}^{\prime}+\mathbf{B D}^{\prime} & =0
\end{aligned}
$$

where $I$ is a $3 \times 3$ unit matrix. These matrix equations lead to a system of equations on $\left(a_{i}, b_{i}, c_{i}, d_{i}, a_{i}^{\prime}, b_{i}^{\prime}, c_{i}^{\prime}, d_{i}^{\prime}\right)$, with $(i=1,2,3)$, which is invariant for permutation of the indexes $\{1,2,3\}$. This property of index permutation invariance allows an extension of some results. Let us denote:

$$
\eta_{1}=d_{1} d_{1}^{\prime}, \quad \eta_{2}=d_{2} d_{2}^{\prime}, \quad \eta_{3}=d_{3} d_{3}^{\prime}
$$


We can write:

$$
\mathbf{D D}^{\prime}=\mathbf{D}^{\prime} \mathbf{D}=\left[\begin{array}{lll}
\eta_{1} & & \\
& \eta_{2} & \\
& & \eta_{3}
\end{array}\right]
$$

Multiplying both sides of Equation (81) by $\mathbf{D}$ one finds: $\mathbf{B} \mathbf{D}^{\prime} \mathbf{D}=-\mathbf{D} \mathbf{A}^{\prime} \mathbf{D}$, and it can be deduced that $\mathbf{B D}^{\prime} \mathbf{D}$ is symmetric. This symmetry implies the following relations:

$$
\eta_{1} d_{3}=\eta_{2} d_{3}, \quad \eta_{2} d_{1}=\eta_{3} d_{1}, \quad \eta_{3} d_{2}=\eta_{1} d_{2}
$$

Multiplying both sides of $\eta_{1} d_{3}=\eta_{2} d_{3}$ by $d_{3}^{\prime}$ and by using Equation (82), one finds $\eta_{1} \eta_{3}=\eta_{2} \eta_{3}$. The index permutation gives:

$$
\eta_{1} \eta_{2}=\eta_{2} \eta_{3}=\eta_{3} \eta_{1}
$$

This equation implies that either $\eta_{1}=\eta_{2}=\eta_{3}$ or two of the $\eta_{i}$ s are equal to zero and the third one is different. The following general structure of the equations is studied in these two cases. Because of the index permutation invariance, the study of the case in which only one $\eta_{i}$ is different from zero (Case 1) can be restricted to the study of the subcase $\eta_{1} \neq 0$, and $\eta_{2}=\eta_{3}=0$ (Case 1). In the other case (Case 2), the subcases $\eta_{1}=\eta_{2}=\eta_{3} \neq 0$ (Case 2.1) and $\eta_{1}=\eta_{2}=\eta_{3}=0$ (Case 2.2) are distinguished.

\section{Case 1.1}

In this case $\eta_{1} \neq 0$, and $\eta_{2}=\eta_{3}=0$. All equations and assumptions are invariant for index permutation $\{2,3\}$. Equation (84) implies: $d_{2}=d_{3}=0$. Multiplying both sides of Equation (80) by $\mathbf{D}$, one finds: $\mathbf{A} \mathbf{D}^{\prime} \mathbf{D}=-\mathbf{D B}^{\prime} \mathbf{D}$. Then, writing $\left(\mathbf{A D}^{\prime} \mathbf{D}\right)_{21}=-\left(\mathbf{D B}^{\prime} \mathbf{D}\right)_{21}$ one finds: $c_{3} \eta_{1}=d_{2} d_{3}^{\prime} d_{1}=0$, and implies $c_{3}=0$, and by index permutation, $c_{2}=0$. Therefore, the matrices $\mathbf{A}, \mathbf{B}$, and $\mathbf{D}$ have the following expressions:

$$
\begin{aligned}
& \mathbf{A}=\left[\begin{array}{ccc}
a_{1} & 0 & 0 \\
0 & a_{2} & c_{1} \\
0 & c_{1} & a_{3}
\end{array}\right], \quad \mathbf{B}=\left[\begin{array}{ccc}
b_{1} & 0 & 0 \\
0 & b_{2} & -d_{1} \\
0 & -d_{1} & b_{3}
\end{array}\right], \\
& \mathbf{D}=\left[\begin{array}{lll}
d_{1} & & \\
& 0 & \\
& & 0
\end{array}\right], \quad \mathbf{D D}^{\prime}=\left[\begin{array}{lll}
\eta_{1} & \\
& 0 & \\
& & 0
\end{array}\right]
\end{aligned}
$$


$\mathbf{A}^{\prime}$ and $\mathbf{B}^{\prime}$ can be deduced from Equations (86), (78) and (79). Matrix $\mathbf{D}^{\prime}$ can also be determined since its elements are given by the nondiagonal terms of $\mathbf{B}^{\prime}$. One finds that these matrices have the same form as $\mathbf{A}, \mathbf{B}$, and D with:

$$
\begin{aligned}
& a_{1}^{\prime}=\left(\frac{1-2 \eta_{1}}{a_{1}}\right), \quad a_{2}^{\prime}=\left(\frac{a_{3}}{a_{2} a_{3}-c_{1}^{2}}\right), \\
& a_{3}^{\prime}=\left(\frac{a_{2}}{a_{2} a_{3}-c_{1}^{2}}\right), \quad c_{1}^{\prime}=\left(-\frac{c_{1}}{a_{2} a_{3}-c_{1}^{2}}\right) \\
& b_{1}^{\prime}=\left(\frac{1-2 \eta_{1}}{b_{1}}\right), \quad b_{2}^{\prime}=\left(\frac{b_{3}}{b_{2} b_{3}-d_{1}^{2}}\right), \\
& b_{3}^{\prime}=\left(\frac{b_{2}}{b_{2} b_{3}-d_{1}^{2}}\right), \quad d_{1}^{\prime}=\left(\frac{-d_{1}}{b_{2} b_{3}-d_{1}^{2}}\right)
\end{aligned}
$$

The parameters defining $\mathbf{A}, \mathbf{B}$ and $\mathbf{D}$ are not all independent. As a matter of fact, Equation (79) implies $\left(\mathbf{B B}^{\prime}\right)_{22}=1$, and then: $b_{2} b_{2}^{\prime}+d_{1}^{\prime \prime} d_{1}=1$. By substituting in this equation for $b_{2}^{\prime}$ by Equation (88) and using $d_{1}^{\prime} d_{1}=\eta_{1}$, one finds:

$$
d_{1}^{2}=\frac{-\eta_{1} b_{2} b_{3}}{1-\eta_{1}}
$$

Equation (80) implies $\left(\mathbf{A D}^{\prime}+\mathbf{D B}^{\prime}\right)_{11}=0$, and then: $a_{1} d_{1}^{\prime}+d_{1} b_{1}^{\prime}=0$. Multiplication of the two sides by $d_{1}$ yields: $a_{1} \eta_{1}+d_{1}^{2} b_{1}^{\prime}=0$, and substituting for $b_{1}^{\prime}$ by Equation (11) and for $d_{1}^{2}$ by Equation (89) gives:

$$
\frac{a_{1} b_{1}}{b_{2} b_{3}}=\frac{1-2 \eta_{1}}{1-\eta_{1}}
$$

In conclusion, in this case all parameters of $\mathbf{A}, \mathbf{B}, \mathbf{D}, \mathbf{A}^{\prime}, \mathbf{B}^{\prime}, \mathbf{D}^{\prime}$, except for the sign of $d_{1}$ and $d_{1}^{\prime}$, are deduced from $\left(a_{1}, a_{2}, a_{3}, c_{1}, b_{1}, b_{2}, b_{3}\right)$ by Equations (87-90).

\section{Case 2}

In this case:

$$
\eta_{1}=\eta_{2}=\eta_{3}=\eta
$$

and all the equations are invariant for index permutation $\{1,2,3\}$. Equation (83) can be written as:

$$
\mathbf{D}^{\prime} \mathbf{D}=\eta \mathbf{I}
$$


By multiplying the two sides of Equation (80) at the right by $\mathbf{D}$, we find:

$$
\mathbf{D B}^{\prime} \mathbf{D}=-\eta \mathbf{A}
$$

and one then deduces $\operatorname{det} \mathbf{B}^{\prime}(\operatorname{det} \mathbf{D})^{2}=-\eta^{3} \operatorname{det} \mathbf{A}$. Since $\mathbf{A}$ and $\mathbf{B}^{\prime}$ are positivedefinite this relation implies:

$$
\eta \leq 0
$$

Besides, taking into account Equation (92), Equation (79) reads: $\mathbf{B B}^{\prime}=$ $(1-2 \eta) \mathbf{I}$, and implies $\left(\mathbf{B B}^{\prime}\right)_{11}=(1-2 \eta)$, or also $b_{1} b_{1}^{\prime}+d_{3} d_{3}^{\prime}+d_{2} d_{2}^{\prime}=(1-2 \eta)$. Substituting in this equation for $d_{3} d_{3}^{\prime}$ and $d_{2} d_{2}^{\prime}$ by $\eta$, one finds $b_{1} b_{1}^{\prime}=1-4 \eta$. Index permutation leads to:

$$
b_{1} b_{1}^{\prime}=b_{2} b_{2}^{\prime}=b_{3} b_{3}^{\prime}=1-4 \eta
$$

The elements of $\mathbf{B}^{-1}$ can be explicitly expressed in terms of $\mathbf{B}$. In particular one can have:

$$
\left(\mathbf{B}^{-1}\right)_{11}=\frac{b_{2} b_{3}-d_{1}^{2}}{\delta}, \quad\left(\mathbf{B}^{-1}\right)_{13}=\frac{d_{2} d_{3}-b_{1} d_{1}}{\delta}
$$

where:

$$
\delta=\operatorname{det} \mathbf{B}=b_{1} b_{2} b_{3}-\left(b_{1} d_{1}^{2}+b_{2} d_{2}^{2}+b_{3} d_{3}^{2}\right)-2 d_{1} d_{2} d_{3}
$$

Using Equation (19) and $\mathbf{B}^{\prime}=(1-2 \eta) \mathbf{B}^{-1}$, one finds:

$$
\begin{aligned}
& b_{1}^{\prime}=(1-2 \eta)\left(\frac{b_{2} b_{3}-d_{1}^{2}}{\delta}\right) \\
& d_{1}^{\prime}=-(1-2 \eta)\left(\frac{d_{2} d_{3}-b_{1} d_{1}}{\delta}\right)
\end{aligned}
$$

Multiplying both sides of Equation (98) by $b_{1}$, one finds $b_{1} b_{1}^{\prime}=(1-2 \eta)$ $\left(b_{1} b_{2} b_{3}-b_{1} d_{1}^{2}\right) / \delta$ and then using Equation (95) one finds:

$$
b_{1} d_{1}^{2}=b_{1} b_{2} b_{3}-\delta \frac{(1-4 \eta)}{(1-2 \eta)}
$$

Note:

$$
b=b_{1} b_{2} b_{3}
$$


Index permutation invariance in Equation (100) implies $b_{1} d_{1}^{2}=b_{2} d_{2}^{2}=b_{3} d_{3}^{2}$. One can define $\kappa$ by writing:

$$
b_{1} d_{1}^{2}=b_{2} d_{2}^{2}=b_{3} d_{3}^{2}=b \kappa
$$

Multiplying the two sides of Equation (99) by $d_{1}$ and using $d_{1} d_{1}^{\prime}=\eta$ and $b_{1} d_{1}^{2}=b \kappa$, one finds:

$$
d_{1} d_{2} d_{3}=-b \kappa-\frac{\delta \eta}{(1-2 \eta)}
$$

Substituting in Equation (97) by Equations (100) and (101), one finds:

$$
\delta=b(1-3 \kappa)-2 d_{1} d_{2} d_{3}
$$

Elimination of $\delta$ and $d_{1} d_{2} d_{3}$ between Equations (104) and (103) leads to:

$$
\begin{aligned}
\delta & =b(1-\kappa)\left(\frac{1-2 \eta}{1-4 \eta}\right) \\
d_{1} d_{2} d_{3} & =-b\left[\frac{\eta+\kappa(1-5 \eta)}{(1-4 \eta)}\right]
\end{aligned}
$$

Equation (102) implies $b^{3} \kappa^{3}=b_{1} b_{2} b_{3} d_{1}^{2} d_{2}^{2} d_{3}^{2}=b\left(d_{1} d_{2} d_{3}\right)^{2}$. Substituting in this equation for $d_{1} d_{2} d_{3}$ by Equation (106), one finds for $\kappa$ the equation: $(1-\kappa)\left[(1-4 \eta)^{2} \kappa^{2}+(2-9 \eta) \eta \kappa+\eta^{2}\right]=0$. The assumption that $\mathbf{B}$ is positivedefinite implies that $\delta>0$ and also $\mathrm{b}_{1}>0, \mathrm{~b}_{2}>0, \mathrm{~b}_{3}>0$, and thus $\mathrm{b}>0$. Then, since $\eta \leq 0$, (28) implies $1-\kappa>0$, the equation on $\kappa$ reduces to:

$$
(1-4 \eta)^{2} \kappa^{2}+(2-9 \eta) \eta \kappa+\eta^{2}=0
$$

Now, consider the two subcases $\eta \neq 0$ (Case 2.1) and $\eta=0$ (Case 2.2).

\section{Case 2.1}

In this case $\eta<0$. It can be deduced from Equation (93) that $\eta \mathbf{A}_{11}=-\left(\mathbf{D B}^{\prime} \mathbf{D}\right)_{11}$, and then $a_{1}=-d_{1}^{2} b_{1}^{\prime} / \eta$. By multiplying the two sides by $b_{1}^{2}$ and by taking account of Equations (95) and (102), one finds $a_{1} b_{1}^{2}=-b \kappa(1-4 \eta) / \eta$. Index permutation invariance then gives:

$$
a_{1} b_{1}^{2}=a_{2} b_{2}^{2}=a_{3} b_{3}^{2}=\frac{-b \kappa(1-4 \eta)}{\eta}
$$


This equation allows one to write $-[b \kappa(1-4 \eta) / \eta]^{3}=a_{1} b_{1}^{2} a_{2} b_{2}^{2} a_{3} b_{3}^{2}=$ $a_{1} a_{2} a_{3} b^{2}$ and to deduce:

$$
b=\beta^{3} a_{1} a_{2} a_{3}, \quad \beta=\frac{-\eta}{[\kappa(1-4 \eta)]}
$$

From Equation (93) one can also deduce $-\eta \mathbf{A}_{23}=\left(\mathbf{D B}^{\prime} \mathbf{D}\right)_{23}$, and so $\eta c_{1}=d_{2} d_{3} d_{1}^{\prime}$. By multiplying the two sides of this equality by $d_{1}^{2}$ and simplifying by $\eta$, one finds $c_{1} d_{1}^{2}=d_{1} d_{2} d_{3}$. By index permutation and by using Equation (106) one finds:

$$
c_{1} d_{1}^{2}=c_{2} d_{2}^{2}=c_{3} d_{3}^{2}=-b \frac{[\eta+\kappa(1-5 \eta)]}{(1-4 \eta)}
$$

Equation (107) has two negative roots, compatible with the condition $\delta>0$ in Equation (105). Now, one can show that if $\left(a_{1}, a_{2}, a_{3}, \eta\right)$ are given and if one of the roots $\kappa$ of Equation (107) is chosen, then all the parameters of $\mathbf{A}, \mathbf{B}, \mathbf{D}, \mathbf{A}^{\prime}, \mathbf{B}^{\prime}, \mathbf{D}^{\prime}$ can be determined, except for the sign of $d_{\mathrm{i}}$ and $d_{i}^{\prime}$. As a matter of fact, in this case, $b$ can be deduced from Equation (109) and then $b_{\mathrm{i}}$ from Equation (108), $d_{i}$ from Equation (102), and $c_{i}$ from Equation (110). The result is:

$$
\begin{aligned}
& b_{1}=\beta \sqrt{a_{2} a_{3}}, \quad b_{2}=\beta \sqrt{a_{1} a_{3}}, \quad b_{3}=\beta \sqrt{a_{1} a_{2}} \\
& d_{1}^{2}=\beta^{2} \kappa a_{1} \sqrt{a_{2} a_{3}}, \quad d_{2}^{2}=\beta^{2} \kappa a_{2} \sqrt{a_{1} a_{3}}, \quad d_{3}^{2}=\beta^{2} \kappa a_{3} \sqrt{a_{1} a_{2}} \\
& c_{1}=\gamma \sqrt{a_{2} a_{3}}, \quad c_{2}=\gamma \sqrt{a_{1} a_{3}}, \quad c_{3}=\gamma \sqrt{a_{1} a_{2}}, \quad \gamma=\beta^{2}(1-\kappa)-\beta
\end{aligned}
$$

$\mathbf{A}^{\prime}$ and $\mathbf{B}^{\prime}$ are given by $\mathbf{A}^{\prime}=(1-2 \eta) \mathbf{A}^{-1}, \mathbf{B}^{\prime}=(1-2 \eta) \mathbf{B}^{-1}$ and $\mathbf{D}^{\prime}$ is deduced from the elements of $\mathbf{B}^{\prime}$.

\section{Case 2.2}

In this case $\eta=0$. Then Equation (107) implies $\kappa=0$, and since $\mathbf{B}$ is positive-definite, Equation (102) implies $d_{1}=d_{2}=d_{3}=0$. This condition and Equation (81) lead to $\mathbf{D}^{\prime}=0$. Therefore, in this case, $\mathbf{A}$ and $\mathbf{B}$ are two independent matrices, $\mathbf{A}^{\prime}=\mathbf{A}^{-1}, \mathbf{B}^{\prime}=\mathbf{B}^{-1}$ and:

$$
\mathbf{D}=\mathbf{D}^{\prime}=0
$$




\section{REFERENCES}

Alliche, A. (2004). Damage Model for Fatigue Loading of Concrete, Int. J. Fatigue, 26: 915-921.

Benitez, F.G. and Rosakis, A.J. (1987). Three-dimensional Elastostatics of a Layer and a Layered Medium, J. Elasticity, 18: 3-50.

Boehler, J.-P. (1975). Contribution théorique et expérimentale à l'étude des milieux plastiques anisotropes, Thèse d'état Institut de Mécanique de Grenoble, France.

Boehler, J.-P. (1982). Comportement mécanique des solides anisotropes, Colloque Euromech 115, Villard-de-Lans, Juin 1979, Editions CNRS, Paris.

Cazzani, A. and Marco, Rovati (2003). Extrema of Young's Modulus for Cubic and Transversely Isotropic Solids, Int. J. Solids and Structures, 40: 1713-1744.

Cazzani, A. and Marco, Rovati (2005). Extrema of Young's Modulus for Elastic Solids with Tetragonal Symmetry, Int. J. Solids and Structures (in press).

Chiarelli, A.S., Shao, J.F. and Hoteit, N. (2003). Modeling of Elastoplastic Damage Behaviour of a Claystone, Int. J. Plasticity, 19: 23-45.

Cowin, S.C. and Mehrabadi, M.M. (1987). On the Identification of Material Symmetry for Anisotropic Elastic Materials, Quart. J. Mech. Appl. Math., 40: 451-476.

Cowin, S.C. and Mehrabadi, M.M. (1995). Anisotropic Symmetries of Linear Elasticity, Appl. Mech. Rev., 48: 247-285.

Daley, P.F. and Hron, F. (1979). Reflection and Transmission Coefficients for Seismic Waves in Ellipsoidally Anisotropic Media, Geophysics, 44: 27-38.

Dragon, A., Halm, D. and Désoyer, Th. (2000). Anisotropic Damage in Quasi-brittle Solids: Modelling Computational Issues and Applications, Comput. Methods Appl. Mech. Engrg., 183: $331-352$.

Halm, D. and Dragon, A. (1988). An Anisotropic Model of Damage and Frictional Sliding for Brittle Materials, Eur. J. Mech., A/Solids, 17(3): 439-460.

Hé, Q.C. and Curnier, A. (1995). A More Fundamental Approach to Damaged Elastic StressStrain Relations, Int. J. Solids Structures, 32(10): 1433-1457.

Hefny, A.M. and Lo, K.L. (1999). Analytical Solutions for Stresses and Displacements around Tunnels Driven in Cross-anisotropic Rocks, Int. J. Numer. Anal. Meth. Geomech., 23: $161-177$.

Homand, F., Morel, E., Henry, J.-P., Cuxac, P. and Hammade, E. (1993). Characterization of the Moduli of Elasticity of an Anisotropic Rock using Dynamic and Static Methods, Int. J. Rock Mech. Min. Sci. \& Geomech. Abstr., 30(5): 527-535.

Kachanov, M. (1992). Effective Elastic Properties of Cracked Solids: Critical Review of Some Concepts, Appl. Mech. Review, 45(8): 304-335.

Lekhnitskii, S.G. (1963). Theory of Elasticity of an Anisotropic Elastic Body, Holden Day Series in Mathematical Physics, Holden-Day, San-Francisco.

Louis, L., Robion, Ph. and David, Ch. (2004). A Single Method for Inversion of Anisotropic Data Sets with Application to Structural Studies, J. Structural Geology, 26: 2065-2072.

Milgrom, M. and Shtrikman, S. (1992). The Energy of Inclusions in Linear Media, Exact Shape Independent Relations, J. Mech. Phys. Solids, 40(5): 927-937.

Milton, G.W. (2002). The Theory of Composites, Cambridge University Press, pp. 145-147.

Min, K.B. and Jing, L. (2003). Numerical Determination of the Equivalent Elastic Compliance Tensor for Fractured Rock Masses using the Distinct Element Method, Int. J. Rock Mech. Min. Sci., 40: 795-816. 
Pan, E. and Amadei, B. (1996). Water Reservoir Loading of Long Anisotropic Valleys with Irregular Topographies, Appl. Math. Modelling, 20: 909-924.

Pan, Y.-C. and Chou, T.-W. (1976). Point Force Solution for an Infinite Transversely Isotropic Solid, Transactions of the ASME, Journal of Applied Mechanics, pp 608-612.

Peres Rodrigues, F. and Aires-Barros, L. (1970). Anisotropy of Endogenetic Rocks-correlation between Micropetrographic Index, Ultimate Strength and Modulus of Elasticity Ellipsoids, Proc. 2nd Congress of the ISRM, Belgrad, pp. 1-23.

Pouya, A. 2000. A Transformation of the Problem of Linear Elastic Structure for Application to Inclusion Problem and to Green Functions, Comptes-Rendus de l'Académie des Sciences de Paris, t. 328, Série II b, pp. 437-443.

Pouya A. and Reiffsteck, Ph. (2003). Analytical Solutions for Foundations on Anisotropic Elastic Soils, In: Proceedings of International Symposium FONDSUP, Presses des Ponts et Chaussées, Paris.

Pouya A. and Zaoui, A. (2005). A Transformation of Elastic Boundary Value Problems with Application to Anisotropic Behavior, I. J. Solids Structures (in press).

Rongved, L. (1955). Force Interior to One of Two Joined Semi-infinite Solids, Proc. 2nd Midwestern Conf. Solid Mechanics, pp. 1-13.

Saint Venant, B. (de) (1863). Sur la distribution des élasticités autour de chaque point d'un solide ou d'un milieu de contexture quelconque, particulièrement lorsqu'il est amorphe sans être isotrope, Journal de Math. Pures et Appliquées, Tome VIII ( $2^{\mathrm{ème}}$ série) pp. 257-430.

Sirotine, Y. and Chaskolskaia, M. (1984). Fondements de la physique des cristaux, Editions MIR, Moscou.

Stroh, A.N. (1958). Dislocations and Cracks in Anisotropic Elasticity, Phil. Mag., 3: 625-646.

Takemura, T., Golshani, A., Oda, M. and Suzuki, K. (2003). Preferred Orientations of Open Microcracks in Granite and their Relation with Anisotropic Elasticity, Int. J. Rock Mech. \& Mininig Sci., 40: 443-454.

Thomsen, L. (1986). Weak Elastic Anisotropy, Geophysics, 51(10): 1954-1966.

Ting, T.C.T. (1996). Anisotropic elasticity, Oxford University Press, Oxford.

Zheng, Q.S. 1997. A Unified Invariant Description of Micromechanically-based Effective Elastic Properties for Two-dimensional Damaged Solids, Mechanics of materials, 25: 273-289. 